Supplementary Information for:

\title{
Exploring Solvent-related Reactions and Corresponding Band-gap Tuning Strategies for Carbon Nanodots based on Solvothermal Synthesis
}

Yu Wang ${ }^{a}$, Yanqi Li ${ }^{a, b}$, and Liang Feng ${ }^{a *}$

a Department of Instrumentation and Analytical Chemistry, CAS Key Laboratory of Separation Science for Analytical Chemistry

Dalian Institute of Chemical Physics, Chinese Academy of Sciences

Dalian 116023, P. R. China

$b$ University of Chinese Academy of Sciences

Beijing 100049, P. R. China 


\section{Contents}

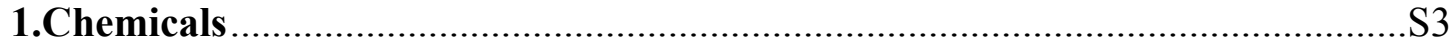

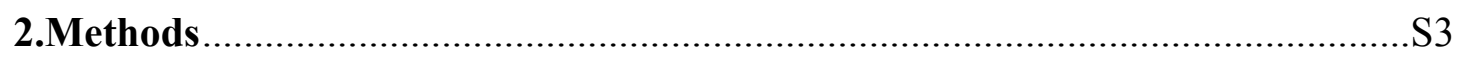

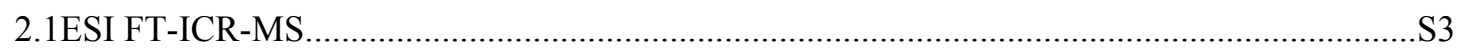

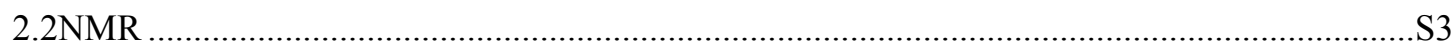

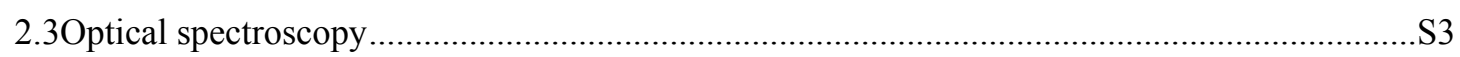

2.4IR spectroscopy ………………………………………………………………....

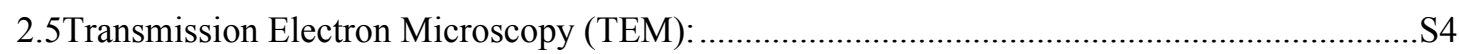

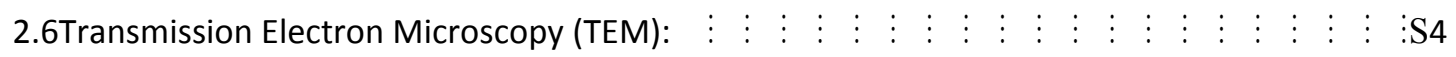

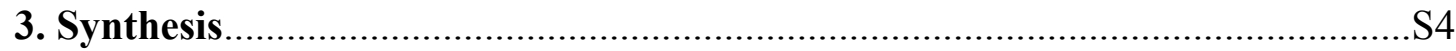

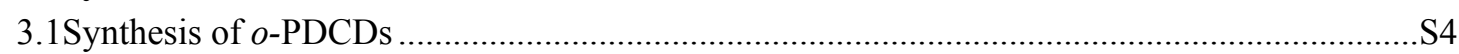

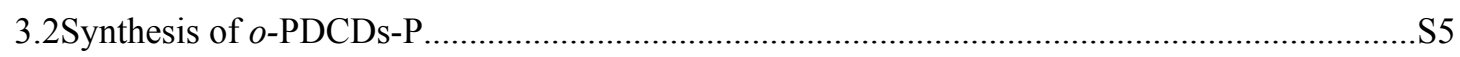

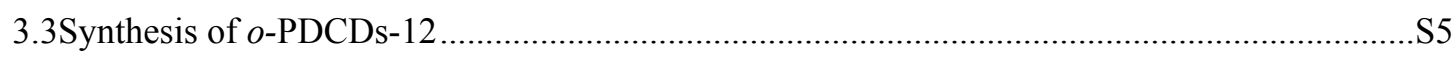

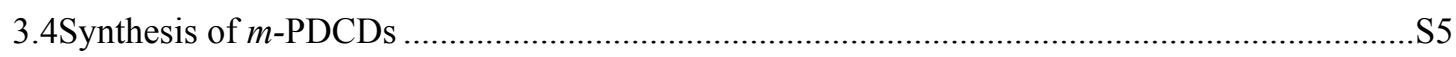

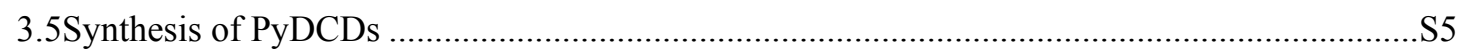

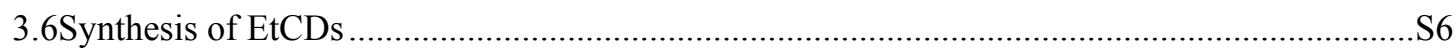

3.7Synthesis of Ru-PDCDs ………………………………………………………......

3.8Synthesis of 2-methylbenzimidazole ……………………………………………………….....

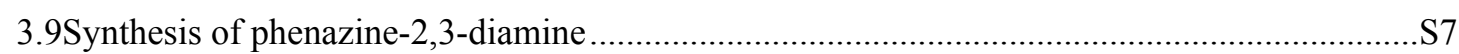

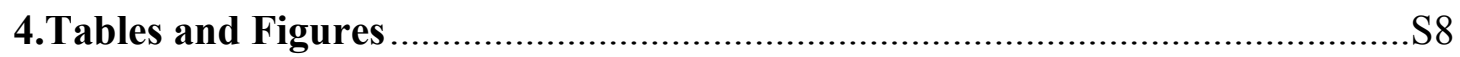

Table S1. The FT-ICR-MS list of the featured fragments of 2,5-PyDCDs (positive mode)...........S8

Table S2. The FT-ICR-MS list of the featured fragments of 2,5-PyDCDs (negative mode)........S10

Table S3 The FT-ICR-MS list of the featured fragments of $m$-PDCDs (positive mode)...............S11

Table S4. The FT-ICR-MS list of the featured fragments of $m$-PDCDs (negative mode).............S11

Table S5. The FT-ICR-MS list of the featured fragments of $o$-PDCDs (positive mode)..............S12

Table S6. The FT-ICR-MS list of the featured fragments of $o$-PDCDs (negative mode)..............S14

Table S7. The FT-ICR-MS list of the featured fragments of EtCDs PyDCDs-P (positive mode)S15

Table S8. The FT-ICR-MS list of the featured fragments of 2,5-PyDCDs-P (positive mode) .....S16

Table S9. The FT-ICR-MS list of the featured fragments of $m$-PDCDs (positive mode)..............S18

Table S10. The FT-ICR-MS list of the featured fragments of $o$-PDCDs-P (positive mode) ........S19

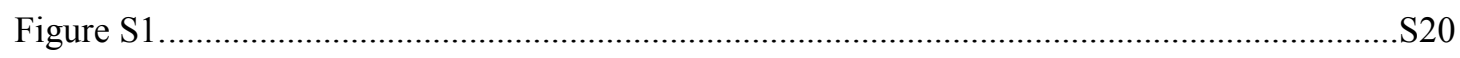

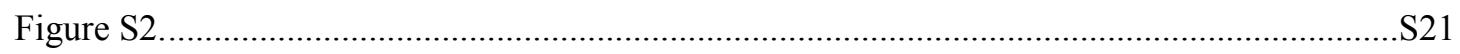

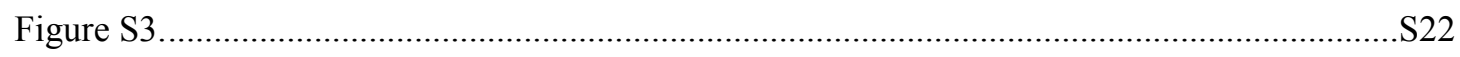

Figure S4

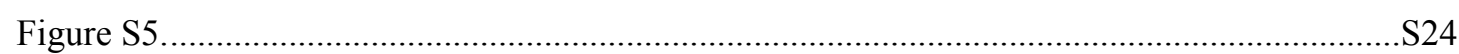

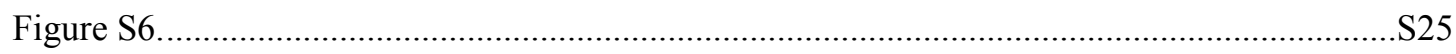

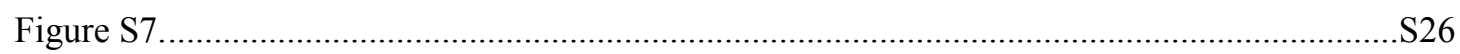

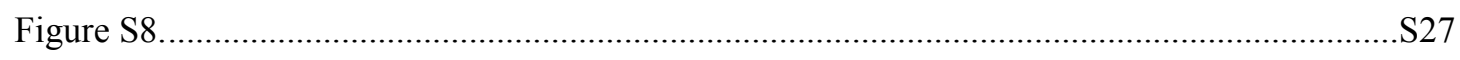

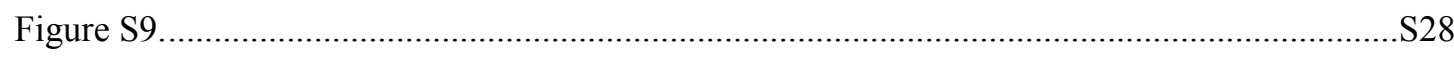




\section{Chemicals}

$m$-phenylenediamine ( $m$-PD), o-phenylenediamine( $o$-PD), 2,5-diaminopyridine (2,5-PyD), 3,4-diaminopyridine (3,4-PyD), 3,5-diaminopyridine (3,5-PyD), 2,4diaminopyridine (2,4-PyD), terephthalic acid, 2,5-dibromoterephthalic acid, biphenyl4,4 =dicarboxylic acid, 4,4'-biphenyldicarboxylic acid (BPDCA), 2,5dibromoterephthalic acid (DBPTA) ethanol and polyethylene glycol (PEG-400) were purchased from Aladdin Reagent (Shanghai) Co., Ltd. 2,2'-bipyridine-4,4'-dicarboxylic acid (BPyDCA), 2,3-diaminopyridine(2,3-PyD), 2,6-diaminopyridine(2,6-PyD) ruthenium trichloride $\left(\mathrm{RuCl}_{3}\right)$ and polyvinyl alcohol (PVA, 86-89\% hydrolyzed) were purchased from Alfa Aesar. Dimethyl sulfoxide- $d 6$ (DMSO- $d 6)$ was purchased from Sigma-Aldrich. All chemicals were used without further purification.

\section{Methods}

\subsection{ESI FT-ICR-MS}

Molecular characterization was performed by a Fourier transform ion cyclotron resonance mass spectrometry (FT-ICR-MS) (Solarix XR, Bruker Daltonics, Bremen, Germany) equipped with a 15 Tesla superconducting magnet. The diluted sodium formate solution at the concentration of $0.1 \mathrm{mg} / \mathrm{ml}$ was used for spectral calibration. The flow rate of syringe pump was $2.0 \mu \mathrm{l} / \mathrm{s}$, and capillary voltage of ESI source was 3500 $\mathrm{V}$ and $4500 \mathrm{~V}$ for positive and negative ion mode, respectively. Ions accumulated in the hexapole and collision cell for $0.1 \mathrm{~ms}$, and transferred into the ICR cell with a flight time of $0.7 \mathrm{~ms}$. The average number of scan time for positive and negative ion mode mass spectra were 16 and 8 . The mass range for all spectra was set to $\mathrm{m} / \mathrm{z}$ 92-1500.

\subsection{NMR}

NMR-spectra were recorded on a Bruker AVAVCE III HD 700MHz spectrometer. Chemicals shifts $(\delta)$ are quoted in ppm downfield of tetramethylsilane. The residual solvent signals were used as references for ${ }^{1} \mathrm{H}$ and HSQC NMR spectra (DMSO-d6: $\delta \mathrm{H}$ $=2.50 \mathrm{ppm}, \delta \mathrm{C}=39.52 \mathrm{ppm})$.

\subsection{Optical spectroscopy}


UV-Vis spectra were recorded at room temperature on Persee TU-1901 UV-Vis spectrophotometer. Fluorescence spectra were recorded on a F-4600 (HITACHI) fluorescence spectrometer. All the spectra were recorded at room temperature using 10 mm path-length cuvettes. Fourier-transform infrared spectroscopy was performed by the attenuated total reflection (ATR) method on a GladiATR (Pike Technologies) equipped with a germanium crystal plate.

\subsection{IR spectroscopy}

Fourier-transform infrared spectroscopy was performed by the attenuated total reflection (ATR) method on a Nicolet iS50 (Thermo Scientific) equipped with a diamond plate and $\mathrm{ZnSe}$ lens.

\subsection{Transmission Electron Microscopy (TEM):}

TEM samples were prepared by dispersing as-synthesized PDCDs in ethanol, sonicating for $10 \mathrm{~min}$, and then drop-casting on to carbon coated copper grids. Samples were then imaged on a JEM-2100 (JEOL) TEM at an acceleration voltage of $200 \mathrm{kV}$. The particles size distribution was calculated and measured by ImageJ software.

\subsection{Photoluminescence Quantum Yield (TEM):}

The absolute fluorescence quantum yields were measured using a Horiba PTI QuantaMaster 400 steady-state fluorescence system with an integrated sphere under ambient conditions.

\section{Synthesis}

\subsection{Synthesis of $o$-PDCDs}

To a $20 \mathrm{~mL}$ glass vial, $0.2 \mathrm{mmol}$ BPyDCA and $1.6 \mathrm{mmol} o$-PD were added into 10 $\mathrm{mL}$ ethanol and sonicating for 10 minutes. The mixture was then transferred to a $50 \mathrm{ml}$ Teflon-sealed autoclave. The autoclave was heated to $200^{\circ} \mathrm{C}$ and kept for 2 hours. After reaction, the autoclave was cooled down to room temperature. The product was transferred to $10 \mathrm{~mL}$ dialysis kit (0.5-1 kDa molecular weight cut off, Float-A-Lyzer G2, Spectrum) and dialyzed against distilled water in $2 \mathrm{~L}$ beaker for 48 hours (changing water for every 12 hours). The as-obtained product was then separated by centrifugation 
at $10000 \mathrm{rpm}(11952 \mathrm{~g})$ for 10 minutes. The supernatant was further purified by filtering with $0.22 \mu \mathrm{m}$ filter membrane (Polyesthersulfone) and then collected as $o$-PDCDs

\subsection{Synthesis of $o$-PDCDs-12}

To a $20 \mathrm{~mL}$ glass vial, $0.2 \mathrm{mmol}$ BPyDCA and $1.6 \mathrm{mmol} o$-PD were added into 10 $\mathrm{mL}$ ethanol and sonicating for 10 minutes. The mixture was then transferred to a $50 \mathrm{ml}$ Teflon-sealed autoclave. The autoclave was heated to $200{ }^{\circ} \mathrm{C}$ and kept for 12 hours. After reaction, the autoclave was cooled down to room temperature. The purification process of raw product was the same as that in the $o$-PDCDs synthesis.

\subsection{Synthesis of $m$-PDCDs}

To a $20 \mathrm{~mL}$ glass vial, $0.1 \mathrm{mmol}$ BPyDCA and $0.4 \mathrm{mmol} m$-PD were added into 10 $\mathrm{mL}$ ethanol and sonicating for 10 minutes. The mixture was then transferred to a $50 \mathrm{ml}$ Teflon-sealed autoclave. The autoclave was heated to $200^{\circ} \mathrm{C}$ and kept for 4 hours. After the reaction, the autoclave was cooled down to the room temperature. The purification process of raw product was the same as that in the $o$-PDCDs synthesis.

\subsection{Synthesis of PyDCDs}

All PyDCDs shared a same synthetic procedure. Specifically, to a $20 \mathrm{~mL}$ glass vial, 0.2 mmol ACDAs (BPyDCA, DBPTA or PTA) and 0.4 mmol PyDs were added into $10 \mathrm{~mL}$ ethanol and sonicating for 10 minutes. The mixture was then transferred to a 50 $\mathrm{ml}$ Teflon-sealed autoclave. The autoclave was heated to $200^{\circ} \mathrm{C}$ and kept for 4 hours. After the reaction, the autoclave was cooled down to the room temperature. The purification process of raw product was the same as that in the $o$-PDCDs synthesis.

\subsection{Synthesis of PDCDs-P}

All PDCDs-P were synthesized by the same procedures with corresponding PDCDs only except the absence of ADCAs.

\subsection{Synthesis of EtCDs}

To a $50 \mathrm{ml}$ Teflon-sealed autoclave, $10 \mathrm{~mL}$ EtOH was added. The autoclave was heated to $200{ }^{\circ} \mathrm{C}$ and kept for 4 hours. After the reaction, the autoclave was cooled down to the room temperature. The purification process of raw product was the same as that in the PDCDs synthesis. 


\subsection{Synthesis of o-PDCDs-Ru}

To a $20 \mathrm{~mL}$ glass vial, $0.2 \mathrm{mmol}$ BPyDCA and $0.2 \mathrm{mmol} o$-PD were added into 10 $\mathrm{mL}$ ethanol and sonicating for 10 minutes. The mixture was then transferred to a $50 \mathrm{ml}$ Teflon-sealed autoclave. The autoclave was heated to $200^{\circ} \mathrm{C}$ and kept for 2 hours. After the reaction, the autoclave was cooled down to the room temperature. $0.1 \mathrm{mmol} \mathrm{RuCl}_{3}$ was added to the product which was then sonicated for 10 minutes. Afterwards, the autoclave was sealed and heated to $200^{\circ} \mathrm{C}$ for another 2 hours . reaction to gain raw oPDCDs-Ru. The purification process of raw o-PDCDs-Ru product was the same as that in the $o$-PDCDs synthesis.

\subsection{Synthesis of DBTPA-m-PD, DBTPA-o-PD, TPA-m-PD, TPA-o-PD, BPDCA-- $m$-PD, BPDCA-o-PD}

DBTPA- $m$-PD, DBTPA- $o$-PD, TPA- $m$-PD, TPA- $o$-PD, BPDCA-- $m$-PD, BPDCA$o$-PD were synthesized by same procedures with those of $m$-PDCDs and $o$-PDCDs, except the replacement of BPyDCA by DBTPA, TPA and BPDCA, respectively.

\subsection{Synthesis of 2-methylbenzimidazole}

To a $25 \mathrm{ml}$ round flask, $1 \mathrm{mmol} o$-PD was dissolved in $6.6 \mathrm{ml}$ acetic acid. After stirring for 10 minutes, $3.4 \mathrm{ml}$ concentrated hydrochloric acid (approximate 12M) was added dropwise. The mixture was refluxed for 2 hours and cooled down to room temperature. The product was dried by rotary evaporation to give grayish pink crystal. The crystal was dissolved in $10 \mathrm{ml}$ distilled water and extracted by $20 \mathrm{ml}$ ethyl acetate for three times. The aqueous phase was obtained and dried by rotary evaporation. After drying, the raw product was purified by column using acetonitrile and distilled water $(10: 1)$ as the eluent. The 2-methylbenzimidazole was obtained as a powder with a paleyellow color. ${ }^{1} \mathrm{H}$ NMR: (700 MHz, DMSO-d6) $\delta$ 7.42-7.45 (dd, 2H), 7.11-7.09 (dd, 2H), 2.50 (s, ).UPLC-MS (ESI): Calculated for $\left[\mathrm{C}_{8} \mathrm{H}_{8} \mathrm{~N}_{2}\right]^{+}: 133.0765$; found: 133.0765

\subsection{Synthesis of phenazine-2,3-diamine}

To a $25 \mathrm{ml}$ round flask, $1 \mathrm{mmol} o$-PD was dissolved in $10 \mathrm{ml} \mathrm{n}$-butanol. The mixture was refluxed for 4 hours and cooled down to room temperature. The product was dried by rotary evaporation to give brownish yellow powder. The powder was purified by 
column using acetonitrile and distilled water (40:1) as the eluent. ${ }^{1} \mathrm{H}$ NMR: (700 MHz, DMSO-d6) $\delta$ 7.90-7.88 (dd, 2H), 7.56-7.54 (dd, 2H), 6.90 (s, 2H), 6.25(s, 4H). UPLCMS (ESI): Calculated for $\left[\mathrm{C}_{12} \mathrm{H}_{10} \mathrm{~N}_{4}\right]^{+}: 211.0983$; found: 211.0969 


\section{Tables and Figures}

Table S1. The FT-ICR-MS list of the featured fragments of 2,5-PyDCDs (positive mode)

\begin{tabular}{|c|c|c|c|c|c|}
\hline $\begin{array}{l}\text { Fragment } \\
\text { Species }\end{array}$ & $\begin{array}{c}\text { Experimental } \\
\text { molecular } \\
\text { weight } \\
(\mathrm{m} / \mathrm{z})\end{array}$ & $\begin{array}{c}\text { Molecular } \\
\text { formula }\end{array}$ & $\begin{array}{c}\text { Calculated } \\
\text { molecular } \\
\text { weight } \\
(\mathrm{m} / \mathrm{z})\end{array}$ & $\begin{array}{l}\text { Error } \\
(\mathrm{ppm})\end{array}$ & $\begin{array}{l}\text { Suggested } \\
\text { structure }\end{array}$ \\
\hline 25-PyD & 110.07125 & $\mathrm{C}_{3} \mathrm{H}_{8} \mathrm{~N}_{3}$ & 110.07127 & 0.19 & $25-\mathrm{PyD}+\mathrm{H}$ \\
\hline 25-PyD & 138.10262 & $\mathrm{C}_{7} \mathrm{H}_{12} \mathrm{~N}_{3}$ & 138.10257 & -0.33 & {$\left[(25-\mathrm{PyD})+\left(\mathrm{C}_{2} \mathrm{H}_{4}\right)\right]+\mathrm{H}$} \\
\hline 25-PyD & 154.09750 & $\mathrm{C}_{7} \mathrm{H}_{12} \mathrm{~N}_{3} \mathrm{O}$ & 154.09749 & -0.08 & {$\left[(25-\mathrm{PyD})+\left(\mathrm{C}_{2} \mathrm{H}_{4} \mathrm{O}\right)\right]+\mathrm{H}$} \\
\hline 25-PyD & 164.11826 & $\mathrm{C}_{9} \mathrm{H}_{14} \mathrm{~N}_{3}$ & 164.11822 & -0.20 & {$\left[(25-\mathrm{PyD})+\left(\mathrm{C}_{2} \mathrm{H}_{4}\right)_{2}-2 \mathrm{H}\right]+\mathrm{H}$} \\
\hline EtOH & 175.09791 & $\mathrm{C}_{8} \mathrm{H}_{15} \mathrm{O}_{4}$ & 175.09649 & -8.15 & {$\left[\left(\mathrm{C}_{2} \mathrm{H}_{4} \mathrm{O}\right)_{4}-2 \mathrm{H}\right]+\mathrm{H}$} \\
\hline 25-PyD & 186.10277 & $\mathrm{C}_{11} \mathrm{H}_{12} \mathrm{~N}_{3}$ & 186.10257 & -1.04 & {$\left[(25-\mathrm{PyD})+\left(\mathrm{C}_{2} \mathrm{H}_{4}\right)_{3}-8 \mathrm{H}\right]+\mathrm{H}$} \\
\hline 25-PyD & 200.08198 & $\mathrm{C}_{11} \mathrm{H}_{10} \mathrm{~N}_{3} \mathrm{O}$ & 200.08184 & -0.73 & {$\left[(25-\mathrm{PyD})+\left(\mathrm{C}_{2} \mathrm{H}_{4}\right)_{2}+\left(\mathrm{C}_{2} \mathrm{H}_{4} \mathrm{O}\right)-10 \mathrm{H}\right]+\mathrm{H}$} \\
\hline 25-PyD & 208.14452 & $\mathrm{C}_{11} \mathrm{H}_{18} \mathrm{~N}_{3} \mathrm{O}$ & 208.14452 & -0.41 & {$\left[(25-\mathrm{PyD})+\left(\mathrm{C}_{2} \mathrm{H}_{4}\right)_{2}+\left(\mathrm{C}_{2} \mathrm{H}_{4} \mathrm{O}\right)-2 \mathrm{H}\right]+\mathrm{H}$} \\
\hline 25-PyD & 214.13401 & $\mathrm{C}_{13} \mathrm{H}_{16} \mathrm{~N}_{3}$ & 214.13387 & -0.63 & {$\left[(25-\mathrm{PyD})+\left(\mathrm{C}_{2} \mathrm{H}_{4}\right)_{4}-8 \mathrm{H}\right]+\mathrm{H}$} \\
\hline EtOH & 217.10470 & $\mathrm{C}_{10} \mathrm{H}_{17} \mathrm{O}_{5}$ & 217.10705 & 10.84 & {$\left[\left(\mathrm{C}_{2} \mathrm{H}_{4} \mathrm{O}\right)_{5}-4 \mathrm{H}\right]+\mathrm{H}$} \\
\hline BPyDCA & 233.06701 & $\mathrm{C}_{15} \mathrm{H}_{9} \mathrm{~N}_{2} \mathrm{O}$ & 233.07094 & 16.86 & {$\left[\left(\mathrm{BPyDCA}-\mathrm{CO}_{2}-\mathrm{H}_{2} \mathrm{O}\right)+\left(\mathrm{C}_{2} \mathrm{H}_{4}\right)_{2}-6 \mathrm{H}\right]+\mathrm{H}$} \\
\hline 25-PyD & 241.11979 & $\mathrm{C}_{12} \mathrm{H}_{13} \mathrm{~N}_{6}$ & 241.11962 & -0.71 & {$\left[(25-\mathrm{PyD})_{2}+\left(\mathrm{C}_{2} \mathrm{H}_{4}\right)-6 \mathrm{H}\right]+\mathrm{H}$} \\
\hline BPyDCA & 245.05576 & $\mathrm{C}_{12} \mathrm{H}_{9} \mathrm{~N}_{2} \mathrm{O}_{4}$ & 245.05568 & -0.32 & $\mathrm{BPyDCA}+\mathrm{H}$ \\
\hline EtOH & 261.13112 & $\mathrm{C}_{12} \mathrm{H}_{21} \mathrm{O}_{6}$ & 261.13326 & 8.20 & {$\left[\left(\mathrm{C}_{2} \mathrm{H}_{4} \mathrm{O}\right)_{6}-4 \mathrm{H}\right]+\mathrm{H}$} \\
\hline 25-PyD & 267.13547 & $\mathrm{C}_{14} \mathrm{H}_{15} \mathrm{~N}_{6}$ & 267.13527 & -0.73 & {$\left[(25-\mathrm{PDA})_{2}+\left(\mathrm{C}_{2} \mathrm{H}_{4}\right)_{2}-8 \mathrm{H}\right]+\mathrm{H}$} \\
\hline BPyDCA & 273.08712 & $\mathrm{C}_{14} \mathrm{H}_{13} \mathrm{~N}_{2} \mathrm{O}_{4}$ & 273.08598 & -0.50 & {$\left[\mathrm{BPyDCA}+\left(\mathrm{C}_{2} \mathrm{H}_{4}\right)\right]+\mathrm{H}$} \\
\hline BPyDCA & 277.10488 & $\mathrm{C}_{17} \mathrm{H}_{13} \mathrm{~N}_{2} \mathrm{O}_{2}$ & 277.09715 & -27.87 & {$\left[\left(\mathrm{BPyDCA}-\mathrm{CO}_{2}-\mathrm{H}_{2} \mathrm{O}\right)+\left(\mathrm{C}_{2} \mathrm{H}_{4}\right)_{2}-6 \mathrm{H}\right]+\left(\mathrm{C}_{2} \mathrm{H}_{4} \mathrm{O}\right)+\mathrm{H}$} \\
\hline 25-PyD & 285.14621 & $\mathrm{C}_{14} \mathrm{H}_{17} \mathrm{~N}_{6} \mathrm{O}$ & 285.14584 & -0.99 & {$\left[(25-\mathrm{PyD})_{2}+\left(\mathrm{C}_{2} \mathrm{H}_{4}\right)+\left(\mathrm{C}_{2} \mathrm{H}_{4} \mathrm{O}\right)-8 \mathrm{H}\right]+\mathrm{H}$} \\
\hline 25-PyD & 291.13540 & $\mathrm{C}_{16} \mathrm{H}_{15} \mathrm{~N}_{6}$ & 291.13527 & -0.43 & {$\left[(25-\mathrm{PDA})_{2}+\left(\mathrm{C}_{2} \mathrm{H}_{4}\right)_{3}-12 \mathrm{H}\right]+\mathrm{H}$} \\
\hline BPyDCA & 295.14426 & $\mathrm{C}_{18} \mathrm{H}_{19} \mathrm{~N}_{2} \mathrm{O}_{2}$ & 295.14410 & -0.52 & {$\left[\left(\mathrm{BPyDCA}-2 \mathrm{CO}_{2}\right)+\left(\mathrm{C}_{2} \mathrm{H}_{4}\right)_{2}+\left(\mathrm{C}_{2} \mathrm{H}_{4} \mathrm{O}\right)_{2}-6 \mathrm{H}\right]+\mathrm{H}$} \\
\hline EtOH & 305.15722 & $\mathrm{C}_{14} \mathrm{H}_{25} \mathrm{O}_{7}$ & 305.15948 & 7.41 & {$\left[\left(\mathrm{C}_{2} \mathrm{H}_{4} \mathrm{O}\right)_{7}-4 \mathrm{H}\right]+\mathrm{H}$} \\
\hline BPyDCA & 321.12106 & $\mathrm{C}_{19} \mathrm{H}_{17} \mathrm{~N}_{2} \mathrm{O}_{3}$ & 321.12337 & 7.20 & {$\left[\left(\mathrm{BPyDCA}-\mathrm{CO}_{2}-\mathrm{H}_{2} \mathrm{O}\right)+\left(\mathrm{C}_{2} \mathrm{H}_{4}\right)_{2}-6 \mathrm{H}\right]+\left(\mathrm{C}_{2} \mathrm{H}_{4} \mathrm{O}\right)_{2}+\mathrm{H}$} \\
\hline EtOH & 349.18343 & $\mathrm{C}_{16} \mathrm{H}_{29} \mathrm{O}_{8}$ & 349.18569 & 6.47 & {$\left[\left(\mathrm{C}_{2} \mathrm{H}_{4} \mathrm{O}\right)_{8}-4 \mathrm{H}\right]+\mathrm{H}$} \\
\hline 25-PyD & 364.15186 & $\mathrm{C}_{17} \mathrm{H}_{22} \mathrm{~N}_{3} \mathrm{O}_{6}$ & 364.15031 & -4.25 & {$\left[(25-\mathrm{PyD})+\left(\mathrm{C}_{2} \mathrm{H}_{4} \mathrm{O}\right)_{6}-10 \mathrm{H}\right]+\mathrm{H}$} \\
\hline
\end{tabular}




\begin{tabular}{|c|c|c|c|c|}
\hline BPyDCA & 365.15766 & $\mathrm{C}_{21} \mathrm{H}_{21} \mathrm{~N}_{2} \mathrm{O}_{4}$ & 365.14958 & $-22.12\left[\left(\mathrm{BPyDCA}-\mathrm{CO}_{2}-\mathrm{H}_{2} \mathrm{O}\right)+\left(\mathrm{C}_{2} \mathrm{H}_{4}\right)_{2}-6 \mathrm{H}\right]+\left(\mathrm{C}_{2} \mathrm{H}_{4} \mathrm{O}\right)_{3}+\mathrm{H}$ \\
\hline 25-PyD & 372.16861 & $\mathrm{C}_{19} \mathrm{H}_{18} \mathrm{~N}_{9}$ & 372.16797 & {$\left[(25-\mathrm{PyD})_{3}+\left(\mathrm{C}_{2} \mathrm{H}_{4}\right)_{2}-12 \mathrm{H}\right]+\mathrm{H}$} \\
\hline EtOH & 393.20963 & $\mathrm{C}_{18} \mathrm{H}_{33} \mathrm{O}_{9}$ & 393.21191 & {$\left[\left(\mathrm{C}_{2} \mathrm{H}_{4} \mathrm{O}\right)_{9}-4 \mathrm{H}\right]+\mathrm{H}$} \\
\hline 25-PyD & 400.19948 & $\mathrm{C}_{21} \mathrm{H}_{22} \mathrm{~N}_{9}$ & 400.19927 & {$\left[(25-\mathrm{PyD})_{3}+\left(\mathrm{C}_{2} \mathrm{H}_{4}\right)_{3}-12 \mathrm{H}\right]+\mathrm{H}$} \\
\hline BPyDCA & 409.18359 & $\mathrm{C}_{23} \mathrm{H}_{25} \mathrm{~N}_{2} \mathrm{O}_{5}$ & 409.17580 & $-19.04\left[\left(\mathrm{BPyDCA}-\mathrm{CO}_{2}-\mathrm{H}_{2} \mathrm{O}\right)+\left(\mathrm{C}_{2} \mathrm{H}_{4}\right)_{2}-6 \mathrm{H}\right]+\left(\mathrm{C}_{2} \mathrm{H}_{4} \mathrm{O}\right)_{4}+\mathrm{H}$ \\
\hline 25-PyD & 416.19474 & $\mathrm{C}_{21} \mathrm{H}_{22} \mathrm{~N}_{9} \mathrm{O}$ & 416.19418 & {$\left[(25-\mathrm{PyD})_{3}+\left(\mathrm{C}_{2} \mathrm{H}_{4}\right)_{2}+\left(\mathrm{C}_{2} \mathrm{H}_{4} \mathrm{O}\right)-12 \mathrm{H}\right]+\mathrm{H}$} \\
\hline EtOH & 437.23631 & $\mathrm{C}_{20} \mathrm{H}_{37} \mathrm{O}_{10}$ & 437.23812 & {$\left[\left(\mathrm{C}_{2} \mathrm{H}_{4} \mathrm{O}\right)_{10}-4 \mathrm{H}\right]+\mathrm{H}$} \\
\hline BPyDCA & 453.20983 & $\mathrm{C}_{25} \mathrm{H}_{29} \mathrm{~N}_{2} \mathrm{O}_{6}$ & 453.20201 & $-17.26\left[\left(\mathrm{BPyDCA}-\mathrm{CO}_{2}-\mathrm{H}_{2} \mathrm{O}\right)+\left(\mathrm{C}_{2} \mathrm{H}_{4}\right)_{2}-6 \mathrm{H}\right]+\left(\mathrm{C}_{2} \mathrm{H}_{4} \mathrm{O}\right)_{5}+\mathrm{H}$ \\
\hline EtOH & 481.26260 & $\mathrm{C}_{22} \mathrm{H}_{41} \mathrm{O}_{11}$ & 481.26434 & {$\left[\left(\mathrm{C}_{2} \mathrm{H}_{4} \mathrm{O}\right)_{11}-4 \mathrm{H}\right]+\mathrm{H}$} \\
\hline BPyDCA & 497.23650 & $\mathrm{C}_{27} \mathrm{H}_{33} \mathrm{~N}_{2} \mathrm{O}_{7}$ & 497.22823 & $-16.64\left[\left(\mathrm{BPyDCA}-\mathrm{CO}_{2}-\mathrm{H}_{2} \mathrm{O}\right)+\left(\mathrm{C}_{2} \mathrm{H}_{4}\right)_{2}-6 \mathrm{H}\right]+\left(\mathrm{C}_{2} \mathrm{H}_{4} \mathrm{O}\right)_{6}+\mathrm{H}$ \\
\hline EtOH & 525.28871 & $\mathrm{C}_{24} \mathrm{H}_{45} \mathrm{O}_{12}$ & 525.29055 & {$\left[\left(\mathrm{C}_{2} \mathrm{H}_{4} \mathrm{O}\right)_{12}-4 \mathrm{H}\right]+\mathrm{H}$} \\
\hline BPyDCA & 541.26260 & $\mathrm{C}_{29} \mathrm{H}_{37} \mathrm{~N}_{2} \mathrm{O}_{8}$ & 541.25444 & $-17.26\left[\left(\mathrm{BPyDCA}-\mathrm{CO}_{2}-\mathrm{H}_{2} \mathrm{O}\right)+\left(\mathrm{C}_{2} \mathrm{H}_{4}\right)_{2}-6 \mathrm{H}\right]+\left(\mathrm{C}_{2} \mathrm{H}_{4} \mathrm{O}\right)_{7}+\mathrm{H}$ \\
\hline EtOH & 569.31499 & $\mathrm{C}_{26} \mathrm{H}_{49} \mathrm{O}_{13}$ & 569.31677 & {$\left[\left(\mathrm{C}_{2} \mathrm{H}_{4} \mathrm{O}\right)_{13}-4 \mathrm{H}\right]+\mathrm{H}$} \\
\hline BPyDCA & 585.28893 & $\mathrm{C}_{31} \mathrm{H}_{41} \mathrm{~N}_{2} \mathrm{O}_{9}$ & 585.28066 & $-14.14\left[\left(\mathrm{BPyDCA}-\mathrm{CO}_{2}-\mathrm{H}_{2} \mathrm{O}+\left(\mathrm{C}_{2} \mathrm{H}_{4}\right)_{2}-6 \mathrm{H}\right]+\left(\mathrm{C}_{2} \mathrm{H}_{4} \mathrm{O}\right)_{8}+\mathrm{H}\right.$ \\
\hline EtOH & 613.34122 & $\mathrm{C}_{28} \mathrm{H}_{53} \mathrm{O}_{14}$ & 613.34298 & {$\left[\left(\mathrm{C}_{2} \mathrm{H}_{4} \mathrm{O}\right)_{14}-4 \mathrm{H}\right]+\mathrm{H}$} \\
\hline BPyDCA & 629.31483 & $\mathrm{C}_{33} \mathrm{H}_{45} \mathrm{~N}_{2} \mathrm{O}_{10}$ & 629.30687 & $-12.65\left[\left(\mathrm{BPyDCA}-\mathrm{CO}_{2}-\mathrm{H}_{2} \mathrm{O}\right)+\left(\mathrm{C}_{2} \mathrm{H}_{4}\right)_{2}-6 \mathrm{H}\right]+\left(\mathrm{C}_{2} \mathrm{H}_{4} \mathrm{O}\right)_{9}+\mathrm{H}$ \\
\hline EtOH & 657.36700 & $\mathrm{C}_{30} \mathrm{H}_{57} \mathrm{O}_{15}$ & 657.36920 & 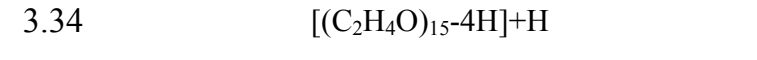 \\
\hline BPyDCA & 673.34130 & $\mathrm{C}_{35} \mathrm{H}_{49} \mathrm{~N}_{2} \mathrm{O}_{11}$ & 673.33309 & $-12.20\left[\left(\mathrm{BPyDCA}-\mathrm{CO}_{2}-\mathrm{H}_{2} \mathrm{O}\right)+\left(\mathrm{C}_{2} \mathrm{H}_{4}\right)_{2}-6 \mathrm{H}\right]+\left(\mathrm{C}_{2} \mathrm{H}_{4} \mathrm{O}\right)_{10}+\mathrm{H}$ \\
\hline EtOH & 701.39320 & $\mathrm{C}_{32} \mathrm{H}_{61} \mathrm{O}_{16}$ & 701.39541 & {$\left[\left(\mathrm{C}_{2} \mathrm{H}_{4} \mathrm{O}\right)_{16}-4 \mathrm{H}\right]+\mathrm{H}$} \\
\hline BPyDCA & 717.36725 & $\mathrm{C}_{37} \mathrm{H}_{53} \mathrm{~N}_{2} \mathrm{O}_{12}$ & 717.35930 & $-11.08\left[\left(\mathrm{BPyDCA}-\mathrm{CO}_{2}-\mathrm{OH}\right)+\left(\mathrm{C}_{2} \mathrm{H}_{4}\right)_{2}-6 \mathrm{H}\right]+\left(\mathrm{C}_{2} \mathrm{H}_{4} \mathrm{O}\right)_{11}+\mathrm{H}$ \\
\hline EtOH & 745.41857 & $\mathrm{C}_{34} \mathrm{H}_{65} \mathrm{O}_{17}$ & 745.42163 & {$\left[\left(\mathrm{C}_{2} \mathrm{H}_{4} \mathrm{O}\right)_{17}-4 \mathrm{H}\right]+\mathrm{H}$} \\
\hline BPyDCA & 761.39416 & $\mathrm{C}_{39} \mathrm{H}_{57} \mathrm{~N}_{2} \mathrm{O}_{13}$ & 761.38552 & $-11.35\left[\left(\mathrm{BPyDCA}-\mathrm{CO}_{2}-\mathrm{OH}\right)+\left(\mathrm{C}_{2} \mathrm{H}_{4}\right)_{2}-6 \mathrm{H}\right]+\left(\mathrm{C}_{2} \mathrm{H}_{4} \mathrm{O}\right)_{12}+\mathrm{H}$ \\
\hline EtOH & 789.44608 & $\mathrm{C}_{36} \mathrm{H}_{69} \mathrm{O}_{18}$ & 789.44784 & {$\left[\left(\mathrm{C}_{2} \mathrm{H}_{4} \mathrm{O}\right)_{18}-4 \mathrm{H}\right]+\mathrm{H}$} \\
\hline BPyDCA & 805.42033 & $\mathrm{C}_{41} \mathrm{H}_{61} \mathrm{~N}_{2} \mathrm{O}_{14}$ & 805.41173 & $-10.67\left[\left(\mathrm{BPyDCA}-\mathrm{CO}_{2}-\mathrm{OH}\right)+\left(\mathrm{C}_{2} \mathrm{H}_{4}\right)_{2}-6 \mathrm{H}\right]+\left(\mathrm{C}_{2} \mathrm{H}_{4} \mathrm{O}\right)_{13}+\mathrm{H}$ \\
\hline EtOH & 833.47148 & $\mathrm{C}_{38} \mathrm{H}_{73} \mathrm{O}_{19}$ & 833.47406 & {$\left[\left(\mathrm{C}_{2} \mathrm{H}_{4} \mathrm{O}\right)_{19}-4 \mathrm{H}\right]+\mathrm{H}$} \\
\hline BPyDCA & 849.44547 & $\mathrm{C}_{43} \mathrm{H}_{65} \mathrm{~N}_{2} \mathrm{O}_{15}$ & 849.43795 & $-8.86\left[\left(\mathrm{BPyDCA}-\mathrm{CO}_{2}-\mathrm{OH}\right)+\left(\mathrm{C}_{2} \mathrm{H}_{4}\right)_{2}-6 \mathrm{H}\right]+\left(\mathrm{C}_{2} \mathrm{H}_{4} \mathrm{O}\right)_{14}+\mathrm{H}$ \\
\hline
\end{tabular}

Table S2. The FT-ICR-MS list of the featured fragments of 2,5-PyDCDs(negative mode)

\begin{tabular}{|c|c|c|c|c|c|}
\hline Fragment & Experimental & Molecular & Calculated & Error & Suggested \\
\hline
\end{tabular}




\begin{tabular}{|c|c|c|c|c|c|}
\hline \multicolumn{3}{|c|}{$\begin{array}{c}\text { Species molecular weight formula } \\
(\mathrm{m} / \mathrm{z})\end{array}$} & \multirow{2}{*}{$\begin{array}{c}\begin{array}{c}\text { molecular } \\
\text { weight } \\
(\mathrm{m} / \mathrm{z})\end{array} \\
199.05130\end{array}$} & \multirow{2}{*}{\begin{tabular}{|c|}
$(\mathrm{ppm})$ \\
-0.74
\end{tabular}} & \multirow{2}{*}{$\begin{array}{c}\text { structure } \\
{\left[\text { BPyDCA-CO } \mathrm{CO}_{2}\right]-\mathrm{H}}\end{array}$} \\
\hline BPyDCA & 199.05145 & $\mathrm{C}_{11} \mathrm{H}_{7} \mathrm{~N}_{2} \mathrm{O}_{2}$ & & & \\
\hline BPyDCA & 243.04134 & $\mathrm{C}_{12} \mathrm{H}_{7} \mathrm{~N}_{2} \mathrm{O}_{4}$ & 243.04113 & -0.32 & BPyDCA-H \\
\hline EtOH & 255.05689 & $\mathrm{C}_{16} \mathrm{H}_{31} \mathrm{O}_{2}$ & 255.23295 & -0.33 & {$\left[(\mathrm{EtOH})_{2}+\left(\mathrm{C}_{2} \mathrm{H}_{4}\right)_{6}-4 \mathrm{H}\right]-\mathrm{H}$} \\
\hline BPyDCA & 265.02321 & $\mathrm{C}_{14} \mathrm{H}_{5} \mathrm{~N}_{2} \mathrm{O}_{4}$ & 265.02548 & 8.57 & {$\left[\mathrm{BPyDCA}+\left(\mathrm{C}_{2} \mathrm{H}_{4}\right)-6 \mathrm{H}\right]-\mathrm{H}$} \\
\hline BPyDCA & 271.07257 & $\mathrm{C}_{14} \mathrm{H}_{11} \mathrm{~N}_{2} \mathrm{O}_{4}$ & 271.07243 & -0.50 & {$\left[\mathrm{BPyDCA}+\left(\mathrm{C}_{2} \mathrm{H}_{4}\right)\right]-\mathrm{H}$} \\
\hline EtOH & 283.26436 & $\mathrm{C}_{18} \mathrm{H}_{35} \mathrm{O}_{2}$ & 283.26425 & -0.38 & {$\left[(\mathrm{EtOH})_{2}+\left(\mathrm{C}_{2} \mathrm{H}_{4}\right)_{7}-4 \mathrm{H}\right]-\mathrm{H}$} \\
\hline BPyDCA & 311.16898 & $\mathrm{C}_{19} \mathrm{H}_{23} \mathrm{~N}_{2} \mathrm{O}_{2}$ & 311.17650 & 24.17 & {$\left[\left(\mathrm{BPyDCA}-\mathrm{CO}_{2}\right)+\left(\mathrm{C}_{2} \mathrm{H}_{4}\right)_{4}\right]-\mathrm{H}$} \\
\hline BPyDCA & 325.18461 & $\mathrm{C}_{20} \mathrm{H}_{25} \mathrm{~N}_{2} \mathrm{O}_{2}$ & 325.19215 & 23.20 & {$\left[\left(\mathrm{BPyDCA}-2 \mathrm{CO}_{2}\right)+\left(\mathrm{C}_{2} \mathrm{H}_{4}\right)_{3}+\left(\mathrm{C}_{2} \mathrm{H}_{4} \mathrm{O}\right)_{2}\right]-\mathrm{H}$} \\
\hline 25-PyD & 370.15376 & $\mathrm{C}_{19} \mathrm{H}_{16} \mathrm{~N}_{9}$ & 370.15342 & -0.93 & {$\left[(25-\mathrm{PyD})_{3}+\left(\mathrm{C}_{2} \mathrm{H}_{4}\right)_{2}-12 \mathrm{H}\right]-\mathrm{H}$} \\
\hline BPyDCA & 487.08989 & $\mathrm{C}_{24} \mathrm{H}_{15} \mathrm{~N}_{4} \mathrm{O}_{8}$ & 487.08954 & -0.72 & $(\mathrm{BPyDCA})_{2}-\mathrm{H}$ \\
\hline BPyDCA & 509.07202 & $\mathrm{C}_{26} \mathrm{H}_{13} \mathrm{~N}_{4} \mathrm{O}_{8}$ & 509.07389 & 3.66 & {$\left[(\mathrm{BPyDCA})_{2}-\left(\mathrm{C}_{2} \mathrm{H}_{4}\right)-6 \mathrm{H}\right]-\mathrm{H}$} \\
\hline BPyDCA & 515.12135 & $\mathrm{C}_{26} \mathrm{H}_{19} \mathrm{~N}_{4} \mathrm{O}_{8}$ & 515.12084 & -1.00 & {$\left[(\mathrm{BPyDCA})_{2}-\left(\mathrm{C}_{2} \mathrm{H}_{4}\right)\right]-\mathrm{H}$} \\
\hline BPyDCA & 537.10351 & $\mathrm{C}_{28} \mathrm{H}_{17} \mathrm{~N}_{4} \mathrm{O}_{8}$ & 537.10519 & 3.13 & {$\left[(\mathrm{BPyDCA})_{2}-\left(\mathrm{C}_{2} \mathrm{H}_{4}\right)_{2}-6 \mathrm{H}\right]-\mathrm{H}$} \\
\hline BPyDCA & 543.15289 & $\mathrm{C}_{28} \mathrm{H}_{23} \mathrm{~N}_{4} \mathrm{O}_{8}$ & 543.15214 & -1.38 & {$\left[(\mathrm{BPyDCA})_{2}-\left(\mathrm{C}_{2} \mathrm{H}_{4}\right)_{2}\right]-\mathrm{H}$} \\
\hline BPyDCA & 573.31898 & $\mathrm{C}_{31} \mathrm{H}_{45} \mathrm{~N}_{2} \mathrm{O}_{8}$ & 573.31814 & -1.47 & {$\left[\left(\mathrm{BPyDCA}-\mathrm{CO}_{2}\right)+\left(\mathrm{C}_{2} \mathrm{H}_{4}\right)_{4}+\left(\mathrm{C}_{2} \mathrm{H}_{4} \mathrm{O}\right)_{6}\right]-\mathrm{H}$} \\
\hline BPyDCA & 601.35019 & $\mathrm{C}_{33} \mathrm{H}_{49} \mathrm{~N}_{2} \mathrm{O}_{8}$ & 601.34944 & -1.24 & {$\left[\left(\mathrm{BPyDCA}-\mathrm{CO}_{2}\right)+\left(\mathrm{C}_{2} \mathrm{H}_{4}\right)_{5}+\left(\mathrm{C}_{2} \mathrm{H}_{4} \mathrm{O}\right)_{6}\right]-\mathrm{H}$} \\
\hline BPyDCA & 629.38169 & $\mathrm{C}_{35} \mathrm{H}_{53} \mathrm{~N}_{2} \mathrm{O}_{8}$ & 629.38074 & -1.50 & {$\left[\left(\mathrm{BPyDCA}-\mathrm{CO}_{2}\right)+\left(\mathrm{C}_{2} \mathrm{H}_{4}\right)_{6}+\left(\mathrm{C}_{2} \mathrm{H}_{4} \mathrm{O}\right)_{6}\right]-\mathrm{H}$} \\
\hline
\end{tabular}

Table S3. The FT-ICR-MS list of the featured fragments of $m$-PDCDs (positive mode)

\begin{tabular}{cccccc}
\hline \hline $\begin{array}{c}\text { Fragment Experimental } \\
\text { molecular } \\
\text { weight } \\
(\mathrm{m} / \mathrm{z})\end{array}$ & $\begin{array}{c}\text { Molecular } \\
\text { formula }\end{array}$ & $\begin{array}{c}\text { Calculated Error } \\
\text { molecular } \\
\text { weight } \\
(\mathrm{m} / \mathrm{z})\end{array}$ & $\begin{array}{c}\text { Suggested } \\
\text { structure }\end{array}$ \\
\hline EtOH & 131.07756 & $\mathrm{C}_{6} \mathrm{H}_{11} \mathrm{O}_{3}$ & 131.07027 & 60.76 & {$\left[\left(\mathrm{C}_{2} \mathrm{H}_{4} \mathrm{O}\right)_{3}-2 \mathrm{H}\right]+\mathrm{H}$} \\
\hline $\boldsymbol{m}$-PD & 133.07654 & $\mathrm{C}_{8} \mathrm{H}_{9} \mathrm{~N}_{2}$ & 133.07602 & -3.85 & {$\left[(m-\mathrm{PD})+\left(\mathrm{C}_{2} \mathrm{H}_{4}\right)-4 \mathrm{H}\right]+\mathrm{H}$} \\
\hline $\boldsymbol{m}$-PD & 135.09173 & $\mathrm{C}_{8} \mathrm{H}_{11} \mathrm{~N}_{2}$ & 135.09167 & -0.38 & {$\left[(m-\mathrm{PD})+\left(\mathrm{C}_{2} \mathrm{H}_{4}\right)-2 \mathrm{H}\right]+\mathrm{H}$} \\
\hline $\boldsymbol{m}$-PD & 159.09180 & $\mathrm{C}_{10} \mathrm{H}_{11} \mathrm{~N}_{2}$ & 159.09167 & -0.77 & $\left.\left[(m-\mathrm{PD})+\left(\mathrm{C}_{2} \mathrm{H}_{4}\right)\right)_{2}-6 \mathrm{H}\right]+\mathrm{H}$ \\
\hline EtOH & 173.08406 & $\mathrm{C}_{8} \mathrm{H}_{13} \mathrm{O}_{4}$ & 173.08084 & -18.61 & {$\left[\left(\mathrm{C}_{2} \mathrm{H}_{4} \mathrm{O}\right)_{4}-4 \mathrm{H}\right]+\mathrm{H}$} \\
\hline EtOH & 175.09791 & $\mathrm{C}_{8} \mathrm{H}_{15} \mathrm{O}_{4}$ & 175.09649 & -8.15 & {$\left[\left(\mathrm{C}_{2} \mathrm{H}_{4} \mathrm{O}\right)_{4}-2 \mathrm{H}\right]+\mathrm{H}$} \\
\hline
\end{tabular}




\begin{tabular}{|c|c|c|c|c|c|}
\hline$m$-PD & 187.12332 & $\mathrm{C}_{12} \mathrm{H}_{15} \mathrm{~N}_{2}$ & 187.12297 & -1.86 & {$\left[(m-\mathrm{PD})+\left(\mathrm{C}_{2} \mathrm{H}_{4}\right)_{3}-6 \mathrm{H}\right]+\mathrm{H}$} \\
\hline$m$-PD & 203.11821 & $\mathrm{C}_{12} \mathrm{H}_{15} \mathrm{~N}_{2} \mathrm{O}$ & 203.11789 & -1.57 & {$\left[(m-\mathrm{PD})+\left(\mathrm{C}_{2} \mathrm{H}_{4}\right)_{2}+\left(\mathrm{C}_{2} \mathrm{H}_{4} \mathrm{O}\right)-6 \mathrm{H}\right]+\mathrm{H}$} \\
\hline EtOH & 217.10486 & $\mathrm{C}_{10} \mathrm{H}_{17} \mathrm{O}_{5}$ & 217.10705 & 10.07 & {$\left[\left(\mathrm{C}_{2} \mathrm{H}_{4} \mathrm{O}\right)_{5}-4 \mathrm{H}\right]+\mathrm{H}$} \\
\hline$m-P D$ & 224.11858 & $\mathrm{C}_{14} \mathrm{H}_{14} \mathrm{~N}_{3}$ & 224.11822 & -1.58 & {$\left[(m-\mathrm{PD})_{2}-\mathrm{NH}+\left(\mathrm{C}_{2} \mathrm{H}_{4}\right)-4 \mathrm{H}\right]+\mathrm{H}$} \\
\hline$m-P D$ & 239.12956 & $\mathrm{C}_{14} \mathrm{H}_{15} \mathrm{~N}_{4}$ & 239.12912 & -1.81 & {$\left[(m-\mathrm{PD})_{2}+\left(\mathrm{C}_{2} \mathrm{H}_{4}\right)-4 \mathrm{H}\right]+\mathrm{H}$} \\
\hline$m-P D$ & 243.16088 & $\mathrm{C}_{14} \mathrm{H}_{19} \mathrm{~N}_{4}$ & 243.16042 & -1.88 & {$\left[(m-\mathrm{PD})_{2}+\left(\mathrm{C}_{2} \mathrm{H}_{4}\right)\right]+\mathrm{H}$} \\
\hline EtOH & 261.13147 & $\mathrm{C}_{12} \mathrm{H}_{21} \mathrm{O}_{6}$ & 261.13326 & 6.86 & {$\left[\left(\mathrm{C}_{2} \mathrm{H}_{4} \mathrm{O}\right)_{6}-4 \mathrm{H}\right]+\mathrm{H}$} \\
\hline$m-P D$ & 265.14290 & $\mathrm{C}_{16} \mathrm{H}_{17} \mathrm{~N}_{4}$ & 265.14477 & -7.08 & {$\left[(m-\mathrm{PD})_{2}+\left(\mathrm{C}_{2} \mathrm{H}_{4}\right)_{2}-8 \mathrm{H}\right]+\mathrm{H}$} \\
\hline$m-P D$ & 269.17661 & $\mathrm{C}_{16} \mathrm{H}_{21} \mathrm{~N}_{4}$ & 269.17607 & -1.98 & {$\left[(m-\mathrm{PD})_{2}+\left(\mathrm{C}_{2} \mathrm{H}_{4}\right)_{2}-4 \mathrm{H}\right]+\mathrm{H}$} \\
\hline BPyDCA & 277.10539 & $\mathrm{C}_{17} \mathrm{H}_{13} \mathrm{~N}_{2} \mathrm{O}_{2}$ & 277.10118 & -15.21 & {$\left[\left(\mathrm{BPyDCA}-\mathrm{CO}_{2}-\mathrm{H}_{2} \mathrm{O}\right)+\left(\mathrm{C}_{2} \mathrm{H}_{4}\right)_{2}-6 \mathrm{H}\right]+\left(\mathrm{C}_{2} \mathrm{H}_{4} \mathrm{O}\right)+\mathrm{H}$} \\
\hline$m-P D$ & 293.17681 & $\mathrm{C}_{18} \mathrm{H}_{21} \mathrm{~N}_{4}$ & 293.17607 & -2.51 & {$\left[(m-\mathrm{PD})_{2}+\left(\mathrm{C}_{2} \mathrm{H}_{4}\right)_{3}-8 \mathrm{H}\right]+\mathrm{H}$} \\
\hline EtOH & 305.15758 & $\mathrm{C}_{14} \mathrm{H}_{25} \mathrm{O}_{7}$ & 305.15567 & 4.75 & {$\left[\left(\mathrm{C}_{2} \mathrm{H}_{4} \mathrm{O}\right)_{7}-4 \mathrm{H}\right]+\mathrm{H}$} \\
\hline$m-P D$ & 319.19234 & $\mathrm{C}_{20} \mathrm{H}_{23} \mathrm{~N}_{4}$ & 319.19172 & -1.93 & {$\left[(m-\mathrm{PD})_{2}+\left(\mathrm{C}_{2} \mathrm{H}_{4}\right)_{4}-10 \mathrm{H}\right]+\mathrm{H}$} \\
\hline BPyDCA & 321.13152 & $\mathrm{C}_{19} \mathrm{H}_{17} \mathrm{~N}_{2} \mathrm{O}_{3}$ & 321.12337 & -25.38 & {$\left[\left(\mathrm{BPyDCA}-\mathrm{CO}_{2}-\mathrm{H}_{2} \mathrm{O}\right)+\left(\mathrm{C}_{2} \mathrm{H}_{4}\right)_{2}-6 \mathrm{H}\right]+\left(\mathrm{C}_{2} \mathrm{H}_{4} \mathrm{O}\right)_{2}+\mathrm{H}$} \\
\hline$m-P D$ & 341.15167 & $\mathrm{C}_{20} \mathrm{H}_{17} \mathrm{~N}_{6}$ & 341.15092 & -2.21 & {$\left[(m-\mathrm{PD})_{3}+\left(\mathrm{C}_{2} \mathrm{H}_{4}\right)-16 \mathrm{H}\right]+\mathrm{H}$} \\
\hline$m-P D$ & 346.16718 & $\mathrm{C}_{20} \mathrm{H}_{20} \mathrm{~N}_{5} \mathrm{O}$ & 346.16624 & -2.73 & {$\left[(m-\mathrm{PD})_{3}-\mathrm{NH}+\left(\mathrm{C}_{2} \mathrm{H}_{4} \mathrm{O}\right)-12 \mathrm{H}\right]+\mathrm{H}$} \\
\hline EtOH & 349.18390 & $\mathrm{C}_{16} \mathrm{H}_{29} \mathrm{O}_{8}$ & 349.18569 & 5.13 & {$\left[\left(\mathrm{C}_{2} \mathrm{H}_{4} \mathrm{O}\right)_{8}-4 \mathrm{H}\right]+\mathrm{H}$} \\
\hline$m-P D$ & 361.17772 & $\mathrm{C}_{20} \mathrm{H}_{21} \mathrm{~N}_{6} \mathrm{O}$ & 361.17714 & -1.61 & {$\left[(m-\mathrm{PD})_{3}+\left(\mathrm{C}_{2} \mathrm{H}_{4} \mathrm{O}\right)-12 \mathrm{H}\right]+\mathrm{H}$} \\
\hline BPyDCA & 365.15821 & $\mathrm{C}_{21} \mathrm{H}_{21} \mathrm{~N}_{2} \mathrm{O}_{4}$ & 365.14958 & -23.61 & {$\left[\left(\mathrm{BPyDCA}-\mathrm{CO}_{2}-\mathrm{H}_{2} \mathrm{O}\right)+\left(\mathrm{C}_{2} \mathrm{H}_{4}\right)_{2}-6 \mathrm{H}\right]+\left(\mathrm{C}_{2} \mathrm{H}_{4} \mathrm{O}\right)_{3}+\mathrm{H}$} \\
\hline$m-P D$ & 370.16711 & $\mathrm{C}_{22} \mathrm{H}_{20} \mathrm{~N}_{5} \mathrm{O}$ & 370.16624 & -2.37 & {$\left[(m-\mathrm{PD})_{3}-\mathrm{NH}+\left(\mathrm{C}_{2} \mathrm{H}_{4} \mathrm{O}\right)-16 \mathrm{H}\right]+\mathrm{H}$} \\
\hline$m-P D$ & 381.29823 & $\mathrm{C}_{24} \mathrm{H}_{37} \mathrm{~N}_{4}$ & 381.30127 & 7.99 & {$\left[(m-\mathrm{PD})_{2}+\left(\mathrm{C}_{2} \mathrm{H}_{4}\right)_{6}-4 \mathrm{H}\right]+\mathrm{H}$} \\
\hline EtOH & 393.21014 & $\mathrm{C}_{18} \mathrm{H}_{33} \mathrm{O}_{9}$ & 393.21191 & 4.50 & {$\left[\left(\mathrm{C}_{2} \mathrm{H}_{4} \mathrm{O}\right)_{9}-4 \mathrm{H}\right]+\mathrm{H}$} \\
\hline$m-P D$ & 399.22769 & $\mathrm{C}_{24} \mathrm{H}_{27} \mathrm{~N}_{6}$ & 399.22917 & 3.72 & {$\left[(m-\mathrm{PD})_{3}+\left(\mathrm{C}_{2} \mathrm{H}_{4}\right)_{3}-10 \mathrm{H}\right]+\mathrm{H}$} \\
\hline BPyDCA & 409.18410 & $\mathrm{C}_{23} \mathrm{H}_{25} \mathrm{~N}_{2} \mathrm{O}_{5}$ & 409.17982 & -10.45 & {$\left[\left(\mathrm{BPyDCA}-\mathrm{CO}_{2}-\mathrm{H}_{2} \mathrm{O}\right)+\left(\mathrm{C}_{2} \mathrm{H}_{4}\right)_{2}-6 \mathrm{H}\right]+\left(\mathrm{C}_{2} \mathrm{H}_{4} \mathrm{O}\right)_{4}+\mathrm{H}$} \\
\hline$m$-PD & 427.26150 & $\mathrm{C}_{26} \mathrm{H}_{31} \mathrm{~N}_{6}$ & 427.26047 & -2.40 & {$\left[(m-\mathrm{PD})_{3}+\left(\mathrm{C}_{2} \mathrm{H}_{4}\right)_{7}-10 \mathrm{H}\right]+\mathrm{H}$} \\
\hline EtOH & 437.23702 & $\mathrm{C}_{20} \mathrm{H}_{37} \mathrm{O}_{10}$ & 437.23812 & 2.53 & {$\left[\left(\mathrm{C}_{2} \mathrm{H}_{4} \mathrm{O}\right)_{10}-4 \mathrm{H}\right]+\mathrm{H}$} \\
\hline BPyDCA & 453.21053 & $\mathrm{C}_{25} \mathrm{H}_{29} \mathrm{~N}_{2} \mathrm{O}_{6}$ & 453.20201 & -18.80 & {$\left[\left(\mathrm{BPyDCA}-\mathrm{CO}_{2}-\mathrm{H}_{2} \mathrm{O}\right)+\left(\mathrm{C}_{2} \mathrm{H}_{4}\right)_{2}-6 \mathrm{H}\right]+\left(\mathrm{C}_{2} \mathrm{H}_{4} \mathrm{O}\right)_{5}+\mathrm{H}$} \\
\hline EtOH & 481.26349 & $\mathrm{C}_{22} \mathrm{H}_{41} \mathrm{O}_{11}$ & 481.26434 & 1.77 & {$\left[\left(\mathrm{C}_{2} \mathrm{H}_{4} \mathrm{O}\right)_{11}-4 \mathrm{H}\right]+\mathrm{H}$} \\
\hline BPyDCA & 497.23738 & $\mathrm{C}_{27} \mathrm{H}_{33} \mathrm{~N}_{2} \mathrm{O}_{7}$ & 497.22823 & -18.40 & {$\left[\left(\mathrm{BPyDCA}-\mathrm{CO}_{2}-\mathrm{H}_{2} \mathrm{O}\right)+\left(\mathrm{C}_{2} \mathrm{H}_{4}\right)_{2}-6 \mathrm{H}\right]+\left(\mathrm{C}_{2} \mathrm{H}_{4} \mathrm{O}\right)_{6}+\mathrm{H}$} \\
\hline
\end{tabular}




\begin{tabular}{|c|c|c|c|c|c|}
\hline EtOH & 525.28981 & $\mathrm{C}_{24} \mathrm{H}_{45} \mathrm{O}_{12}$ & 525.29055 & 1.41 & {$\left[\left(\mathrm{C}_{2} \mathrm{H}_{4} \mathrm{O}\right)_{12}-4 \mathrm{H}\right]+\mathrm{H}$} \\
\hline BPyDCA & 541.26362 & $\mathrm{C}_{29} \mathrm{H}_{37} \mathrm{~N}_{2} \mathrm{O}_{8}$ & 541.25444 & -16.95 & {$\left[\left(\mathrm{BPyDCA}-\mathrm{CO}_{2}-\mathrm{H}_{2} \mathrm{O}\right)+\left(\mathrm{C}_{2} \mathrm{H}_{4}\right)_{2}-6 \mathrm{H}\right]+\left(\mathrm{C}_{2} \mathrm{H}_{4} \mathrm{O}\right)_{7}+\mathrm{H}$} \\
\hline EtOH & 569.31623 & $\mathrm{C}_{26} \mathrm{H}_{49} \mathrm{O}_{13}$ & 569.31677 & 0.95 & {$\left[\left(\mathrm{C}_{2} \mathrm{H}_{4} \mathrm{O}\right)_{13}-4 \mathrm{H}\right]+\mathrm{H}$} \\
\hline BPyDCA & 585.29016 & $\mathrm{C}_{31} \mathrm{H}_{41} \mathrm{~N}_{2} \mathrm{O}_{9}$ & 585.28066 & -16.24 & {$\left[\left(\mathrm{BPyDCA}-\mathrm{CO}_{2}-\mathrm{H}_{2} \mathrm{O}+\left(\mathrm{C}_{2} \mathrm{H}_{4}\right)_{2}-6 \mathrm{H}\right]+\left(\mathrm{C}_{2} \mathrm{H}_{4} \mathrm{O}\right)_{8}+\mathrm{H}\right.$} \\
\hline EtOH & 613.34262 & $\mathrm{C}_{28} \mathrm{H}_{53} \mathrm{O}_{14}$ & 613.34298 & 0.59 & {$\left[\left(\mathrm{C}_{2} \mathrm{H}_{4} \mathrm{O}\right)_{14}-4 \mathrm{H}\right]+\mathrm{H}$} \\
\hline BPyDCA & 629.31483 & $\mathrm{C}_{33} \mathrm{H}_{45} \mathrm{~N}_{2} \mathrm{O}_{10}$ & 629.30687 & -14.70 & {$\left[\left(\mathrm{BPyDCA}-\mathrm{CO}_{2}-\mathrm{H}_{2} \mathrm{O}\right)+\left(\mathrm{C}_{2} \mathrm{H}_{4}\right)_{2}-6 \mathrm{H}\right]+\left(\mathrm{C}_{2} \mathrm{H}_{4} \mathrm{O}\right)_{9}+\mathrm{H}$} \\
\hline EtOH & 657.36840 & $\mathrm{C}_{30} \mathrm{H}_{57} \mathrm{O}_{15}$ & 657.36920 & 1.21 & {$\left[\left(\mathrm{C}_{2} \mathrm{H}_{4} \mathrm{O}\right)_{15^{-}}-\mathrm{H}\right]+\mathrm{H}$} \\
\hline BPyDCA & 673.34278 & $\mathrm{C}_{35} \mathrm{H}_{49} \mathrm{~N}_{2} \mathrm{O}_{11}$ & 673.33309 & -14.39 & {$\left[\left(\mathrm{BPyDCA}-\mathrm{CO}_{2}-\mathrm{H}_{2} \mathrm{O}\right)+\left(\mathrm{C}_{2} \mathrm{H}_{4}\right)_{2}-6 \mathrm{H}\right]+\left(\mathrm{C}_{2} \mathrm{H}_{4} \mathrm{O}\right)_{10}+\mathrm{H}$} \\
\hline EtOH & 701.39541 & $\mathrm{C}_{32} \mathrm{H}_{61} \mathrm{O}_{16}$ & 701.39541 & 1.34 & {$\left[\left(\mathrm{C}_{2} \mathrm{H}_{4} \mathrm{O}\right)_{16^{-}}-4 \mathrm{H}\right]+\mathrm{H}$} \\
\hline BPyDCA & 717.36862 & $\mathrm{C}_{37} \mathrm{H}_{53} \mathrm{~N}_{2} \mathrm{O}_{12}$ & 717.35930 & -12.99 & {$\left[\left(\mathrm{BPyDCA}-\mathrm{CO}_{2}-\mathrm{OH}\right)+\left(\mathrm{C}_{2} \mathrm{H}_{4}\right)_{2}-6 \mathrm{H}\right]+\left(\mathrm{C}_{2} \mathrm{H}_{4} \mathrm{O}\right)_{11}+\mathrm{H}$} \\
\hline EtOH & 745.42066 & $\mathrm{C}_{34} \mathrm{H}_{65} \mathrm{O}_{17}$ & 745.42163 & 1.30 & {$\left[\left(\mathrm{C}_{2} \mathrm{H}_{4} \mathrm{O}\right)_{17}-4 \mathrm{H}\right]+\mathrm{H}$} \\
\hline BPyDCA & 761.39561 & $\mathrm{C}_{39} \mathrm{H}_{57} \mathrm{~N}_{2} \mathrm{O}_{13}$ & 761.38552 & -13.26 & {$\left[\left(\mathrm{BPyDCA}-\mathrm{CO}_{2}-\mathrm{OH}\right)+\left(\mathrm{C}_{2} \mathrm{H}_{4}\right)_{2}-6 \mathrm{H}\right]+\left(\mathrm{C}_{2} \mathrm{H}_{4} \mathrm{O}\right)_{12}+\mathrm{H}$} \\
\hline EtOH & 789.44770 & $\mathrm{C}_{36} \mathrm{H}_{69} \mathrm{O}_{18}$ & 789.44784 & 0.17 & {$\left[\left(\mathrm{C}_{2} \mathrm{H}_{4} \mathrm{O}\right)_{18}-4 \mathrm{H}\right]+\mathrm{H}$} \\
\hline BPyDCA & 805.42241 & $\mathrm{C}_{41} \mathrm{H}_{61} \mathrm{~N}_{2} \mathrm{O}_{14}$ & 805.41173 & -13.26 & {$\left[\left(\mathrm{BPyDCA}-\mathrm{CO}_{2}-\mathrm{OH}\right)+\left(\mathrm{C}_{2} \mathrm{H}_{4}\right)_{2}-6 \mathrm{H}\right]+\left(\mathrm{C}_{2} \mathrm{H}_{4} \mathrm{O}\right)_{13}+\mathrm{H}$} \\
\hline EtOH & 833.47300 & $\mathrm{C}_{38} \mathrm{H}_{73} \mathrm{O}_{19}$ & 833.47406 & 1.26 & {$\left[\left(\mathrm{C}_{2} \mathrm{H}_{4} \mathrm{O}\right)_{19}-4 \mathrm{H}\right]+\mathrm{H}$} \\
\hline BPyDCA & 849.44730 & $\mathrm{C}_{43} \mathrm{H}_{65} \mathrm{~N}_{2} \mathrm{O}_{15}$ & 849.43795 & -11.02 & {$\left[\left(\mathrm{BPyDCA}-\mathrm{CO}_{2}-\mathrm{OH}\right)+\left(\mathrm{C}_{2} \mathrm{H}_{4}\right)_{2}-6 \mathrm{H}\right]+\left(\mathrm{C}_{2} \mathrm{H}_{4} \mathrm{O}\right)_{14}+\mathrm{H}$} \\
\hline
\end{tabular}

Table S4. The FT-ICR-MS list of the featured fragments of $m$-PDCDs (negative mode)

\begin{tabular}{lccccc}
\hline Fragment & $\begin{array}{c}\text { Experimental } \\
\text { Species }\end{array}$ & $\begin{array}{c}\text { Molecular weight } \\
(\mathrm{m} / \mathrm{z})\end{array}$ & $\begin{array}{c}\text { Calculated } \\
\text { formula } \\
\text { weight } \\
(\mathrm{m} / \mathrm{z})\end{array}$ & $\begin{array}{c}\text { Error } \\
(\mathrm{ppm})\end{array}$ & $\begin{array}{c}\text { Suggested } \\
\text { structure }\end{array}$ \\
\hline BPyDCA & 199.05147 & $\mathrm{C}_{11} \mathrm{H}_{7} \mathrm{~N}_{2} \mathrm{O}_{2}$ & 199.05130 & -0.85 & {$\left[\mathrm{BPyDCA}-\mathrm{CO}_{2}\right]-\mathrm{H}$} \\
\hline BPyDCA & 243.04160 & $\mathrm{C}_{12} \mathrm{H}_{7} \mathrm{~N}_{2} \mathrm{O}_{4}$ & 243.04113 & -1.95 & BPyDCA-H \\
\hline EtOH & 255.23326 & $\mathrm{C}_{16} \mathrm{H}_{31} \mathrm{O}_{2}$ & 255.23295 & -1.21 & {$\left[(\mathrm{EtOH})_{2}+\left(\mathrm{C}_{2} \mathrm{H}_{4}\right)_{6}-4 \mathrm{H}\right]-\mathrm{H}$} \\
\hline BPyDCA & 265.02338 & $\mathrm{C}_{14} \mathrm{H}_{5} \mathrm{~N}_{2} \mathrm{O}_{4}$ & 265.02548 & 7.94 & {$\left[\mathrm{BPyDCA}+\left(\mathrm{C}_{2} \mathrm{H}_{4}\right)-6 \mathrm{H}\right]-\mathrm{H}$} \\
\hline BPyDCA & 271.07279 & $\mathrm{C}_{14} \mathrm{H}_{11} \mathrm{~N}_{2} \mathrm{O}_{4}$ & 271.07243 & -1.33 & {$\left[\mathrm{BPyDCA}+\left(\mathrm{C}_{2} \mathrm{H}_{4}\right)\right]-\mathrm{H}$} \\
\hline EtOH & 283.26464 & $\mathrm{C}_{18} \mathrm{H}_{35} \mathrm{O}_{2}$ & 283.26425 & -1.37 & {$\left[(\mathrm{EtOH})_{2}+\left(\mathrm{C}_{2} \mathrm{H}_{4}\right)_{7}-4 \mathrm{H}\right]-\mathrm{H}$} \\
\hline BPyDCA & 311.16960 & $\mathrm{C}_{19} \mathrm{H}_{23} \mathrm{~N}_{2} \mathrm{O}_{2}$ & 311.17650 & 22.19 & $\left.\left[(\mathrm{BPyDCA}-\mathrm{CO})_{2}\right)+\left(\mathrm{C}_{2} \mathrm{H}_{4}\right)_{4}\right]-\mathrm{H}$ \\
\hline BPyDCA & 325.18461 & $\mathrm{C}_{20} \mathrm{H}_{25} \mathrm{~N}_{2} \mathrm{O}_{2}$ & 325.19215 & 23.20 & $\left.\left[(\mathrm{BPyDCA}-2 \mathrm{CO})_{2}\right)+\left(\mathrm{C}_{2} \mathrm{H}_{4}\right)_{3}+\left(\mathrm{C}_{2} \mathrm{H}_{4} \mathrm{O}\right)_{2}\right]-\mathrm{H}$ \\
\hline BPyDCA & 487.09054 & $\mathrm{C}_{24} \mathrm{H}_{15} \mathrm{~N}_{4} \mathrm{O}_{8}$ & 487.08954 & -2.05 & $(\mathrm{BPyDCA})_{2}-\mathrm{H}$ \\
\hline
\end{tabular}




\begin{tabular}{lccccc}
\hline BPyDCA & 509.07280 & $\mathrm{C}_{26} \mathrm{H}_{13} \mathrm{~N}_{4} \mathrm{O}_{8}$ & 509.07389 & 2.13 & {$\left[(\mathrm{BPyDCA})_{2}-\left(\mathrm{C}_{2} \mathrm{H}_{4}\right)-6 \mathrm{H}\right]-\mathrm{H}$} \\
\hline BPyDCA & 515.12218 & $\mathrm{C}_{26} \mathrm{H}_{19} \mathrm{~N}_{4} \mathrm{O}_{8}$ & 515.12084 & -2.61 & {$\left[(\mathrm{BPyDCA})_{2}-\left(\mathrm{C}_{2} \mathrm{H}_{4}\right)\right]-\mathrm{H}$} \\
\hline BPyDCA & 537.10403 & $\mathrm{C}_{28} \mathrm{H}_{17} \mathrm{~N}_{4} \mathrm{O}_{8}$ & 537.10519 & 3.13 & {$\left[(\mathrm{BPyDCA})_{2}-\left(\mathrm{C}_{2} \mathrm{H}_{4}\right)_{2}-6 \mathrm{H}\right]-\mathrm{H}$} \\
\hline BPyDCA & 543.15289 & $\mathrm{C}_{28} \mathrm{H}_{23} \mathrm{~N}_{4} \mathrm{O}_{8}$ & 543.15214 & 2.16 & {$\left[(\mathrm{BPyDCA})_{2}-\left(\mathrm{C}_{2} \mathrm{H}_{4}\right)_{2}\right]-\mathrm{H}$} \\
\hline BPyDCA & 573.31992 & $\mathrm{C}_{31} \mathrm{H}_{45} \mathrm{~N}_{2} \mathrm{O}_{8}$ & 573.31814 & -3.10 & {$\left[\left(\mathrm{BPyDCA}-\mathrm{CO}_{2}\right)+\left(\mathrm{C}_{2} \mathrm{H}_{4}\right)_{4}+\left(\mathrm{C}_{2} \mathrm{H}_{4} \mathrm{O}\right)_{6}\right]-\mathrm{H}$} \\
\hline BPyDCA & 601.35120 & $\mathrm{C}_{33} \mathrm{H}_{49} \mathrm{~N}_{2} \mathrm{O}_{8}$ & 601.34944 & -2.92 & {$\left[\left(\mathrm{BPyDCA}-\mathrm{CO}_{2}\right)+\left(\mathrm{C}_{2} \mathrm{H}_{4}\right)_{5}+\left(\mathrm{C}_{2} \mathrm{H}_{4} \mathrm{O}\right)_{6}\right]-\mathrm{H}$} \\
\hline \hline
\end{tabular}

Table S5. The FT-ICR-MS list of the featured fragments of $o$-PDCDs (positive mode)

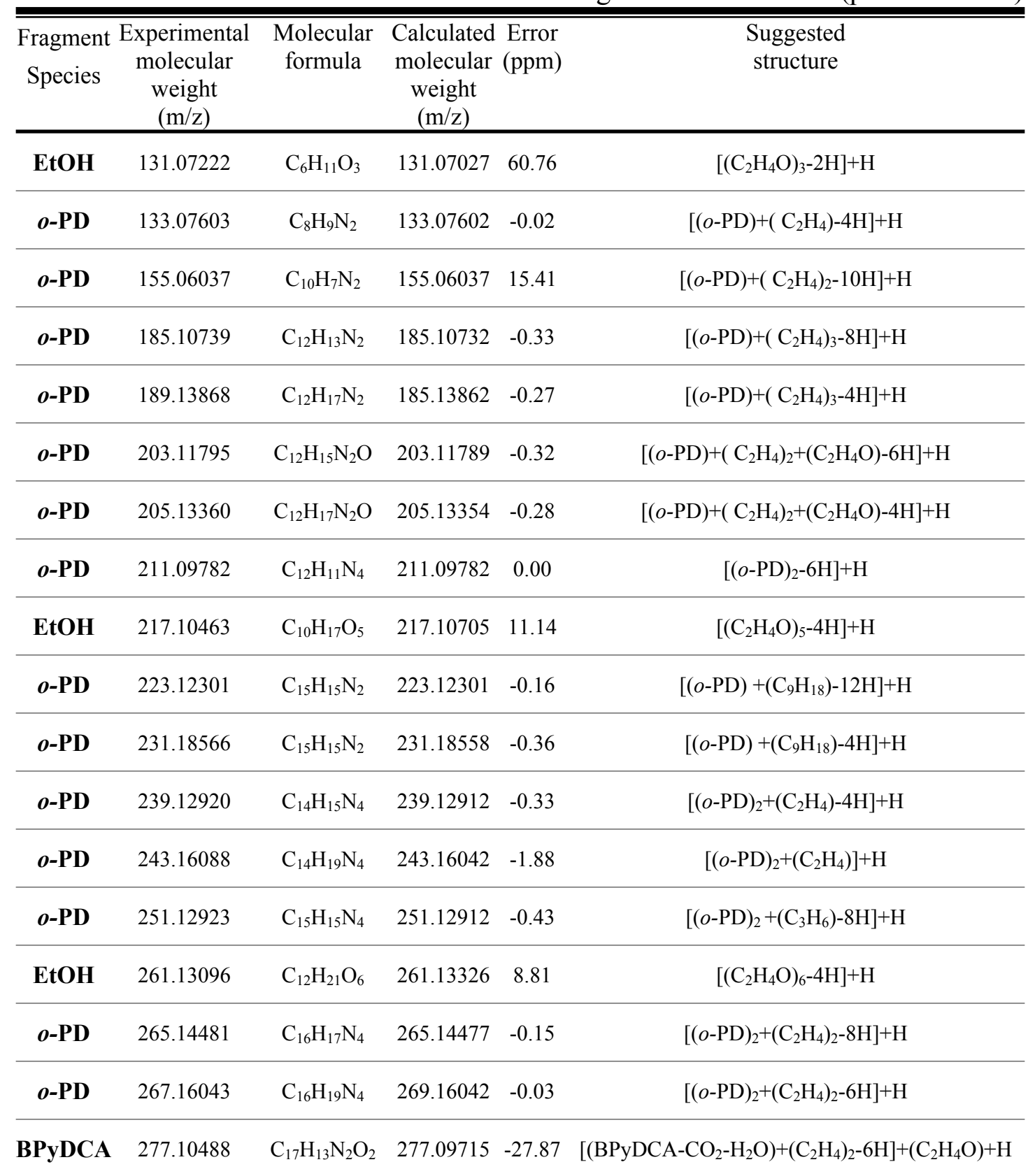




\begin{tabular}{|c|c|c|c|c|c|}
\hline$o-P D$ & 283.15533 & $\mathrm{C}_{16} \mathrm{H}_{19} \mathrm{~N}_{4} \mathrm{O}$ & 283.15534 & 0.02 & {$\left[(o-\mathrm{PD})_{2}+\left(\mathrm{C}_{2} \mathrm{H}_{4}\right)+\left(\mathrm{C}_{2} \mathrm{H}_{4} \mathrm{O}\right)-6 \mathrm{H}\right]+\mathrm{H}$} \\
\hline$o-P D$ & 291.16043 & $\mathrm{C}_{18} \mathrm{H}_{19} \mathrm{~N}_{4}$ & 291.16042 & -0.03 & {$\left[(o-\mathrm{PD})_{2}+\left(\mathrm{C}_{2} \mathrm{H}_{4}\right)_{3}-10 \mathrm{H}\right]+\mathrm{H}$} \\
\hline$o-P D$ & 293.17621 & $\mathrm{C}_{18} \mathrm{H}_{21} \mathrm{~N}_{4}$ & 293.17607 & -0.47 & {$\left[(o-\mathrm{PD})_{2}+\left(\mathrm{C}_{2} \mathrm{H}_{4}\right)_{3}-8 \mathrm{H}\right]+\mathrm{H}$} \\
\hline BPyDCA & 295.06910 & $\mathrm{C}_{16} \mathrm{H}_{11} \mathrm{~N}_{2} \mathrm{O}_{4}$ & 295.07133 & 7.58 & {$\left[(\mathrm{BPyDCA})+\left(\mathrm{C}_{2} \mathrm{H}_{4}\right)_{2}-6 \mathrm{H}\right]+\mathrm{H}$} \\
\hline EtOH & 305.15758 & $\mathrm{C}_{14} \mathrm{H}_{25} \mathrm{O}_{7}$ & 305.15567 & 4.75 & {$\left[\left(\mathrm{C}_{2} \mathrm{H}_{4} \mathrm{O}\right)_{7}-4 \mathrm{H}\right]+\mathrm{H}$} \\
\hline BPyDCA & 321.13152 & $\mathrm{C}_{19} \mathrm{H}_{17} \mathrm{~N}_{2} \mathrm{O}_{3}$ & 321.12337 & -25.38 & {$\left[\left(\mathrm{BPyDCA}-\mathrm{CO}_{2}-\mathrm{H}_{2} \mathrm{O}\right)+\left(\mathrm{C}_{2} \mathrm{H}_{4}\right)_{2}-6 \mathrm{H}\right]+\left(\mathrm{C}_{2} \mathrm{H}_{4} \mathrm{O}\right)_{2}+\mathrm{H}$} \\
\hline$o-P D$ & 341.15094 & $\mathrm{C}_{20} \mathrm{H}_{17} \mathrm{~N}_{6}$ & 341.15092 & -0.05 & {$\left[(o-\mathrm{PD})_{3}+\left(\mathrm{C}_{2} \mathrm{H}_{4}\right)-16 \mathrm{H}\right]+\mathrm{H}$} \\
\hline EtOH & 349.18326 & $\mathrm{C}_{16} \mathrm{H}_{29} \mathrm{O}_{8}$ & 349.18569 & 6.98 & {$\left[\left(\mathrm{C}_{2} \mathrm{H}_{4} \mathrm{O}\right)_{8}-4 \mathrm{H}\right]+\mathrm{H}$} \\
\hline$o-P D$ & 353.17611 & $\mathrm{C}_{18} \mathrm{H}_{21} \mathrm{~N}_{6} \mathrm{O}_{2}$ & 353.17205 & -11.49 & {$\left[(o-\mathrm{PD})_{3}+2\left(\mathrm{H}_{2} \mathrm{O}\right)-8 \mathrm{H}\right]+\mathrm{H}$} \\
\hline BPyDCA & 365.15737 & $\mathrm{C}_{21} \mathrm{H}_{21} \mathrm{~N}_{2} \mathrm{O}_{4}$ & 365.14958 & -21.33 & {$\left[\left(\mathrm{BPyDCA}-\mathrm{CO}_{2}-\mathrm{H}_{2} \mathrm{O}\right)+\left(\mathrm{C}_{2} \mathrm{H}_{4}\right)_{2}-6 \mathrm{H}\right]+\left(\mathrm{C}_{2} \mathrm{H}_{4} \mathrm{O}\right)_{3}+\mathrm{H}$} \\
\hline$o$-PD & 369.18236 & $\mathrm{C}_{22} \mathrm{H}_{21} \mathrm{~N}_{6}$ & 369.18222 & -0.39 & {$\left[(o-\mathrm{PD})_{3}+\left(\mathrm{C}_{2} \mathrm{H}_{4}\right)_{2}-12 \mathrm{H}\right]+\mathrm{H}$} \\
\hline$o-P D$ & 381.29747 & $\mathrm{C}_{24} \mathrm{H}_{37} \mathrm{~N}_{4}$ & 381.29725 & -0.58 & {$\left[(o-\mathrm{PD})_{2}+\left(\mathrm{C}_{2} \mathrm{H}_{4}\right)_{6}-4 \mathrm{H}\right]+\mathrm{H}$} \\
\hline EtOH & 393.20942 & $\mathrm{C}_{18} \mathrm{H}_{33} \mathrm{O}_{9}$ & 393.21191 & 6.34 & {$\left[\left(\mathrm{C}_{2} \mathrm{H}_{4} \mathrm{O}\right)_{9}-4 \mathrm{H}\right]+\mathrm{H}$} \\
\hline$o-P D$ & 397.27149 & $\mathrm{C}_{22} \mathrm{H}_{33} \mathrm{~N}_{6} \mathrm{O}$ & 397.27104 & -1.14 & {$\left[(o-\mathrm{PD})_{3}+\left(\mathrm{C}_{2} \mathrm{H}_{4}\right)+\left(\mathrm{C}_{2} \mathrm{H}_{4} \mathrm{O}\right)\right]+\mathrm{H}$} \\
\hline BPyDCA & 409.18410 & $\mathrm{C}_{23} \mathrm{H}_{25} \mathrm{~N}_{2} \mathrm{O}_{5}$ & 409.17982 & -10.45 & {$\left[\left(\mathrm{BPyDCA}-\mathrm{CO}_{2}-\mathrm{H}_{2} \mathrm{O}\right)+\left(\mathrm{C}_{2} \mathrm{H}_{4}\right)_{2}-6 \mathrm{H}\right]+\left(\mathrm{C}_{2} \mathrm{H}_{4} \mathrm{O}\right)_{4}+\mathrm{H}$} \\
\hline BPyDCA & 413.11281 & $\mathrm{C}_{24} \mathrm{H}_{17} \mathrm{~N}_{2} \mathrm{O}_{5}$ & 413.11454 & 4.17 & $\left.\left[\left(\mathrm{BPyDCA}-\mathrm{H}_{2} \mathrm{O}\right)\right]+\left(\mathrm{C}_{2} \mathrm{H}_{4}\right)_{4}+\left(\mathrm{C}_{2} \mathrm{H}_{4} \mathrm{O}\right)_{2}-14 \mathrm{H}\right]+\mathrm{H}$ \\
\hline EtOH & 437.23582 & $\mathrm{C}_{20} \mathrm{H}_{37} \mathrm{O}_{10}$ & 437.23812 & 5.26 & {$\left[\left(\mathrm{C}_{2} \mathrm{H}_{4} \mathrm{O}\right)_{10}-4 \mathrm{H}\right]+\mathrm{H}$} \\
\hline$o$-PD & 449.24498 & $\mathrm{C}_{28} \mathrm{H}_{29} \mathrm{~N}_{6}$ & 449.24482 & -0.35 & {$\left[(o-\mathrm{PD})_{3}+\left(\mathrm{C}_{2} \mathrm{H}_{4}\right)_{5}-16 \mathrm{H}\right]+\mathrm{H}$} \\
\hline BPyDCA & 453.20948 & $\mathrm{C}_{25} \mathrm{H}_{29} \mathrm{~N}_{2} \mathrm{O}_{6}$ & 453.20201 & -16.48 & {$\left[\left(\mathrm{BPyDCA}-\mathrm{CO}_{2}-\mathrm{H}_{2} \mathrm{O}\right)+\left(\mathrm{C}_{2} \mathrm{H}_{4}\right)_{2}-6 \mathrm{H}\right]+\left(\mathrm{C}_{2} \mathrm{H}_{4} \mathrm{O}\right)_{5}+\mathrm{H}$} \\
\hline EtOH & 481.26207 & $\mathrm{C}_{22} \mathrm{H}_{41} \mathrm{O}_{11}$ & 481.26434 & 4.72 & {$\left[\left(\mathrm{C}_{2} \mathrm{H}_{4} \mathrm{O}\right)_{11}-4 \mathrm{H}\right]+\mathrm{H}$} \\
\hline BPyDCA & 497.23593 & $\mathrm{C}_{27} \mathrm{H}_{33} \mathrm{~N}_{2} \mathrm{O}_{7}$ & 497.22823 & -15.50 & {$\left[\left(\mathrm{BPyDCA}-\mathrm{CO}_{2}-\mathrm{H}_{2} \mathrm{O}\right)+\left(\mathrm{C}_{2} \mathrm{H}_{4}\right)_{2}-6 \mathrm{H}\right]+\left(\mathrm{C}_{2} \mathrm{H}_{4} \mathrm{O}\right)_{6}+\mathrm{H}$} \\
\hline EtOH & 525.28817 & $\mathrm{C}_{24} \mathrm{H}_{45} \mathrm{O}_{12}$ & 525.29055 & 4.54 & {$\left[\left(\mathrm{C}_{2} \mathrm{H}_{4} \mathrm{O}\right)_{12}-4 \mathrm{H}\right]+\mathrm{H}$} \\
\hline BPyDCA & 541.26201 & $\mathrm{C}_{29} \mathrm{H}_{37} \mathrm{~N}_{2} \mathrm{O}_{8}$ & 541.25444 & -13.99 & {$\left[\left(\mathrm{BPyDCA}-\mathrm{CO}_{2}-\mathrm{H}_{2} \mathrm{O}\right)+\left(\mathrm{C}_{2} \mathrm{H}_{4}\right)_{2}-6 \mathrm{H}\right]+\left(\mathrm{C}_{2} \mathrm{H}_{4} \mathrm{O}\right)_{7}+\mathrm{H}$} \\
\hline EtOH & 569.31438 & $\mathrm{C}_{26} \mathrm{H}_{49} \mathrm{O}_{13}$ & 569.31677 & 4.19 & {$\left[\left(\mathrm{C}_{2} \mathrm{H}_{4} \mathrm{O}\right)_{13}-4 \mathrm{H}\right]+\mathrm{H}$} \\
\hline BPyDCA & 585.28824 & $\mathrm{C}_{31} \mathrm{H}_{41} \mathrm{~N}_{2} \mathrm{O}_{9}$ & 585.28066 & -12.95 & {$\left[\left(\mathrm{BPyDCA}-\mathrm{CO}_{2}-\mathrm{H}_{2} \mathrm{O}+\left(\mathrm{C}_{2} \mathrm{H}_{4}\right)_{2}-6 \mathrm{H}\right]+\left(\mathrm{C}_{2} \mathrm{H}_{4} \mathrm{O}\right)_{8}+\mathrm{H}\right.$} \\
\hline EtOH & 613.34059 & $\mathrm{C}_{28} \mathrm{H}_{53} \mathrm{O}_{14}$ & 613.34298 & 3.90 & {$\left[\left(\mathrm{C}_{2} \mathrm{H}_{4} \mathrm{O}\right)_{14}-4 \mathrm{H}\right]+\mathrm{H}$} \\
\hline BPyDCA & 629.31425 & $\mathrm{C}_{33} \mathrm{H}_{45} \mathrm{~N}_{2} \mathrm{O}_{10}$ & 629.30687 & -11.72 & {$\left[\left(\mathrm{BPyDCA}-\mathrm{CO}_{2}-\mathrm{H}_{2} \mathrm{O}\right)+\left(\mathrm{C}_{2} \mathrm{H}_{4}\right)_{2}-6 \mathrm{H}\right]+\left(\mathrm{C}_{2} \mathrm{H}_{4} \mathrm{O}\right)_{9}+\mathrm{H}$} \\
\hline EtOH & 657.366510 & $\mathrm{C}_{30} \mathrm{H}_{57} \mathrm{O}_{15}$ & 657.36920 & 4.08 & {$\left[\left(\mathrm{C}_{2} \mathrm{H}_{4} \mathrm{O}\right)_{15}-4 \mathrm{H}\right]+\mathrm{H}$} \\
\hline BPyDCA & 673.34050 & $\mathrm{C}_{35} \mathrm{H}_{49} \mathrm{~N}_{2} \mathrm{O}_{11}$ & 673.33309 & -11.01 & {$\left[\left(\mathrm{BPyDCA}-\mathrm{CO}_{2}-\mathrm{H}_{2} \mathrm{O}\right)+\left(\mathrm{C}_{2} \mathrm{H}_{4}\right)_{2}-6 \mathrm{H}\right]+\left(\mathrm{C}_{2} \mathrm{H}_{4} \mathrm{O}\right)_{10}+\mathrm{H}$} \\
\hline
\end{tabular}




\begin{tabular}{cccccc}
\hline EtOH & 701.39248 & $\mathrm{C}_{32} \mathrm{H}_{61} \mathrm{O}_{16}$ & 701.39541 & 4.18 & {$\left[\left(\mathrm{C}_{2} \mathrm{H}_{4} \mathrm{O}\right)_{16}-4 \mathrm{H}\right]+\mathrm{H}$} \\
\hline BPyDCA & 717.36667 & $\mathrm{C}_{37} \mathrm{H}_{53} \mathrm{~N}_{2} \mathrm{O}_{12}$ & 717.35930 & -10.27 & {$\left[\left(\mathrm{BPyDCA}-\mathrm{CO}_{2}-\mathrm{OH}\right)+\left(\mathrm{C}_{2} \mathrm{H}_{4}\right)_{2}-6 \mathrm{H}\right]+\left(\mathrm{C}_{2} \mathrm{H}_{4} \mathrm{O}\right)_{11}+\mathrm{H}$} \\
\hline EtOH & 745.41857 & $\mathrm{C}_{34} \mathrm{H}_{65} \mathrm{O}_{17}$ & 745.42163 & 4.10 & {$\left[\left(\mathrm{C}_{2} \mathrm{H}_{4} \mathrm{O}\right)_{17}-4 \mathrm{H}\right]+\mathrm{H}$} \\
\hline BPyDCA & 761.39315 & $\mathrm{C}_{39} \mathrm{H}_{57} \mathrm{~N}_{2} \mathrm{O}_{13}$ & 761.38552 & -10.03 & {$\left[\left(\mathrm{BPyDCA}-\mathrm{CO}_{2}-\mathrm{OH}\right)+\left(\mathrm{C}_{2} \mathrm{H}_{4}\right)_{2}-6 \mathrm{H}\right]+\left(\mathrm{C}_{2} \mathrm{H}_{4} \mathrm{O}\right)_{12}+\mathrm{H}$} \\
\hline EtOH & 789.44501 & $\mathrm{C}_{36} \mathrm{H}_{69} \mathrm{O}_{18}$ & 789.44784 & 3.59 & {$\left[\left(\mathrm{C}_{2} \mathrm{H}_{4} \mathrm{O}\right)_{18}-4 \mathrm{H}\right]+\mathrm{H}$} \\
\hline BPyDCA & 805.41926 & $\mathrm{C}_{41} \mathrm{H}_{61} \mathrm{~N}_{2} \mathrm{O}_{14}$ & 805.41173 & -9.34 & {$\left[\left(\mathrm{BPyDCA}-\mathrm{CO}_{2}-\mathrm{OH}\right)+\left(\mathrm{C}_{2} \mathrm{H}_{4}\right)_{2}-6 \mathrm{H}\right]+\left(\mathrm{C}_{2} \mathrm{H}_{4} \mathrm{O}\right)_{13}+\mathrm{H}$} \\
\hline EtOH & 833.47100 & $\mathrm{C}_{38} \mathrm{H}_{73} \mathrm{O}_{19}$ & 833.47406 & 3.67 & {$\left[\left(\mathrm{C}_{2} \mathrm{H}_{4} \mathrm{O}\right)_{19}-4 \mathrm{H}\right]+\mathrm{H}$} \\
\hline BPyDCA & 849.44520 & $\mathrm{C}_{43} \mathrm{H}_{65} \mathrm{~N}_{2} \mathrm{O}_{15}$ & 849.43795 & -8.54 & {$\left[\left(\mathrm{BPyDCA}-\mathrm{CO}_{2}-\mathrm{OH}\right)+\left(\mathrm{C}_{2} \mathrm{H}_{4}\right)_{2}-6 \mathrm{H}\right]+\left(\mathrm{C}_{2} \mathrm{H}_{4} \mathrm{O}\right)_{14}+\mathrm{H}$} \\
\hline EtOH & 877.49757 & $\mathrm{C}_{40} \mathrm{H}_{77} \mathrm{O}_{20}$ & 8877.50027 & 3.08 & {$\left[\left(\mathrm{C}_{2} \mathrm{H}_{4} \mathrm{O}\right)_{20}-4 \mathrm{H}\right]+\mathrm{H}$} \\
\hline \hline
\end{tabular}

Table S6. The FT-ICR-MS list of the featured fragments of $o$-PDCDs (negative mode)

\begin{tabular}{|c|c|c|c|c|c|}
\hline $\begin{array}{c}\text { Fragment } \\
\text { Species }\end{array}$ & $\begin{array}{l}\text { Experimental } \\
\text { molecular weigh } \\
(\mathrm{m} / \mathrm{z})\end{array}$ & $\begin{array}{l}\text { Molecular } \\
\text { tt formula }\end{array}$ & $\begin{array}{c}\text { Calculated } \\
\text { molecular } \\
\text { weight } \\
(\mathrm{m} / \mathrm{z})\end{array}$ & $\begin{array}{l}\text { Error } \\
(\mathrm{ppm})\end{array}$ & $\begin{array}{l}\text { Suggested } \\
\text { structure }\end{array}$ \\
\hline BPyDCA & 243.04104 & $\mathrm{C}_{12} \mathrm{H}_{7} \mathrm{~N}_{2} \mathrm{O}_{4}$ & 243.04113 & 0.37 & BPyDCA-H \\
\hline EtOH & 255.23323 & $\mathrm{C}_{16} \mathrm{H}_{31} \mathrm{O}_{2}$ & 255.23295 & -1.06 & {$\left[(\mathrm{EtOH})_{2}+\left(\mathrm{C}_{2} \mathrm{H}_{4}\right)_{6}-4 \mathrm{H}\right]-\mathrm{H}$} \\
\hline BPyDCA & 265.02304 & $\mathrm{C}_{14} \mathrm{H}_{5} \mathrm{~N}_{2} \mathrm{O}_{4}$ & 265.02548 & 9.20 & {$\left[\mathrm{BPyDCA}+\left(\mathrm{C}_{2} \mathrm{H}_{4}\right)-6 \mathrm{H}\right]-\mathrm{H}$} \\
\hline BPyDCA & 271.07257 & $\mathrm{C}_{14} \mathrm{H}_{11} \mathrm{~N}_{2} \mathrm{O}_{4}$ & 271.07243 & -0.52 & {$\left[\mathrm{BPyDCA}+\left(\mathrm{C}_{2} \mathrm{H}_{4}\right)\right]-\mathrm{H}$} \\
\hline EtOH & 283.26429 & $\mathrm{C}_{18} \mathrm{H}_{35} \mathrm{O}_{2}$ & 283.26425 & -0.14 & {$\left[(\mathrm{EtOH})_{2}+\left(\mathrm{C}_{2} \mathrm{H}_{4}\right)_{7}-4 \mathrm{H}\right]-\mathrm{H}$} \\
\hline BPyDCA & 311.16865 & $\mathrm{C}_{19} \mathrm{H}_{23} \mathrm{~N}_{2} \mathrm{O}_{2}$ & 311.17650 & 25.24 & {$\left[\left(\mathrm{BPyDCA}-\mathrm{CO}_{2}\right)+\left(\mathrm{C}_{2} \mathrm{H}_{4}\right)_{4}\right]-\mathrm{H}$} \\
\hline BPyDCA & 325.18421 & $\mathrm{C}_{20} \mathrm{H}_{25} \mathrm{~N}_{2} \mathrm{O}_{2}$ & 325.19215 & 24.43 & {$\left[\left(\mathrm{BPyDCA}-2 \mathrm{H}_{2} \mathrm{O}\right)+\left(\mathrm{C}_{2} \mathrm{H}_{4}\right)_{5}-4 \mathrm{H}\right]-\mathrm{H}$} \\
\hline BPyDCA & 487.08944 & $\mathrm{C}_{24} \mathrm{H}_{15} \mathrm{~N}_{4} \mathrm{O}_{8}$ & 487.08954 & 0.19 & $(\mathrm{BPyDCA})_{2}-\mathrm{H}$ \\
\hline BPyDCA & 509.07121 & $\mathrm{C}_{26} \mathrm{H}_{13} \mathrm{~N}_{4} \mathrm{O}_{8}$ & 509.07389 & 5.26 & {$\left[(\mathrm{BPyDCA})_{2}-\left(\mathrm{C}_{2} \mathrm{H}_{4}\right)-6 \mathrm{H}\right]-\mathrm{H}$} \\
\hline BPyDCA & 515.12005 & $\mathrm{C}_{26} \mathrm{H}_{19} \mathrm{~N}_{4} \mathrm{O}_{8}$ & 515.12084 & 1.53 & {$\left[(\mathrm{BPyDCA})_{2}-\left(\mathrm{C}_{2} \mathrm{H}_{4}\right)\right]-\mathrm{H}$} \\
\hline BPyDCA & 537.10208 & $\mathrm{C}_{28} \mathrm{H}_{17} \mathrm{~N}_{4} \mathrm{O}_{8}$ & 537.10519 & 5.78 & {$\left[(\mathrm{BPyDCA})_{2}-\left(\mathrm{C}_{2} \mathrm{H}_{4}\right)_{2}-6 \mathrm{H}\right]-\mathrm{H}$} \\
\hline BPyDCA & 573.31754 & $\mathrm{C}_{31} \mathrm{H}_{45} \mathrm{~N}_{2} \mathrm{O}_{8}$ & 573.31679 & -1.30 & {$\left[\left(\mathrm{BPyDCA}-\mathrm{CO}_{2}\right)+\left(\mathrm{C}_{2} \mathrm{H}_{4}\right)_{4}+\left(\mathrm{C}_{2} \mathrm{H}_{4} \mathrm{O}\right)_{6}\right]-\mathrm{H}$} \\
\hline BPyDCA & 601.34899 & $\mathrm{C}_{33} \mathrm{H}_{49} \mathrm{~N}_{2} \mathrm{O}_{8}$ & 601.34944 & 0.75 & {$\left[\left(\mathrm{BPyDCA}-\mathrm{CO}_{2}\right)+\left(\mathrm{C}_{2} \mathrm{H}_{4}\right)_{5}+\left(\mathrm{C}_{2} \mathrm{H}_{4} \mathrm{O}\right)_{6}\right]-\mathrm{H}$} \\
\hline BPyDCA & 629.37991 & $\mathrm{C}_{35} \mathrm{H}_{53} \mathrm{~N}_{2} \mathrm{O}_{8}$ & 629.38074 & 1.32 & {$\left[\left(\mathrm{BPyDCA}-\mathrm{CO}_{2}\right)+\left(\mathrm{C}_{2} \mathrm{H}_{4}\right)_{6}+\left(\mathrm{C}_{2} \mathrm{H}_{4} \mathrm{O}\right)_{6}\right]-\mathrm{H}$} \\
\hline
\end{tabular}

Table S7. The FT-ICR-MS list of the featured fragments of EtCDs (positive mode) 


\begin{tabular}{lcccll}
\hline \hline Species & $\begin{array}{c}\text { molecular } \\
\text { weight } \\
(\mathrm{m} / \mathrm{z})\end{array}$ & formula & $\begin{array}{c}\text { molecular }(\mathrm{ppm}) \\
\text { weight } \\
(\mathrm{m} / \mathrm{z})\end{array}$ & \multicolumn{1}{c}{ structure } \\
\hline EtOH & 305.15951 & $\mathrm{C}_{14} \mathrm{H}_{25} \mathrm{O}_{7}$ & 305.15948 & -0.11 & {$\left[\left(\mathrm{C}_{2} \mathrm{H}_{4} \mathrm{O}\right)_{7}-4 \mathrm{H}\right]+\mathrm{H}$} \\
\hline EtOH & 349.18577 & $\mathrm{C}_{16} \mathrm{H}_{29} \mathrm{O}_{8}$ & 349.18569 & -0.22 & {$\left[\left(\mathrm{C}_{2} \mathrm{H}_{4} \mathrm{O}\right)_{8}-4 \mathrm{H}\right]+\mathrm{H}$} \\
\hline EtOH & 393.20963 & $\mathrm{C}_{18} \mathrm{H}_{33} \mathrm{O}_{9}$ & 393.21191 & 5.78 & {$\left[\left(\mathrm{C}_{2} \mathrm{H}_{4} \mathrm{O}\right)_{9}-4 \mathrm{H}\right]+\mathrm{H}$} \\
\hline EtOH & 437.23921 & $\mathrm{C}_{20} \mathrm{H}_{37} \mathrm{O}_{10}$ & 437.23812 & -2.48 & {$\left[\left(\mathrm{C}_{2} \mathrm{H}_{4} \mathrm{O}\right)_{10}-4 \mathrm{H}\right]+\mathrm{H}$} \\
\hline EtOH & 481.26472 & $\mathrm{C}_{22} \mathrm{H}_{41} \mathrm{O}_{11}$ & 481.26434 & -0.79 & {$\left[\left(\mathrm{C}_{2} \mathrm{H}_{4} \mathrm{O}\right)_{11}-4 \mathrm{H}\right]+\mathrm{H}$} \\
\hline EtOH & 525.29327 & $\mathrm{C}_{24} \mathrm{H}_{45} \mathrm{O}_{12}$ & 525.29055 & -5.18 & {$\left[\left(\mathrm{C}_{2} \mathrm{H}_{4} \mathrm{O}\right)_{12}-4 \mathrm{H}\right]+\mathrm{H}$} \\
\hline EtOH & 569.32026 & $\mathrm{C}_{26} \mathrm{H}_{49} \mathrm{O}_{13}$ & 569.31677 & -6.13 & {$\left[\left(\mathrm{C}_{2} \mathrm{H}_{4} \mathrm{O}\right)_{13}-4 \mathrm{H}\right]+\mathrm{H}$} \\
\hline EtOH & 613.34761 & $\mathrm{C}_{28} \mathrm{H}_{53} \mathrm{O}_{14}$ & 613.34298 & -7.54 & {$\left[\left(\mathrm{C}_{2} \mathrm{H}_{4} \mathrm{O}\right)_{14}-4 \mathrm{H}\right]+\mathrm{H}$} \\
\hline EtOH & 657.37336 & $\mathrm{C}_{30} \mathrm{H}_{57} \mathrm{O}_{15}$ & 657.36920 & -6.34 & {$\left[\left(\mathrm{C}_{2} \mathrm{H}_{4} \mathrm{O}\right)_{15}-4 \mathrm{H}\right]+\mathrm{H}$} \\
\hline EtOH & 745.42716 & $\mathrm{C}_{34} \mathrm{H}_{65} \mathrm{O}_{17}$ & 745.42163 & -7.42 & {$\left[\left(\mathrm{C}_{2} \mathrm{H}_{4} \mathrm{O}\right)_{17}-4 \mathrm{H}\right]+\mathrm{H}$} \\
\hline EtOH & 789.45654 & $\mathrm{C}_{36} \mathrm{H}_{69} \mathrm{O}_{18}$ & 789.44784 & -11.02 & {$\left[\left(\mathrm{C}_{2} \mathrm{H}_{4} \mathrm{O}\right)_{18}-4 \mathrm{H}\right]+\mathrm{H}$} \\
\hline$\left.\left(\mathrm{C}_{2} \mathrm{H}_{4} \mathrm{O}\right)_{19}-4 \mathrm{H}\right]+\mathrm{H}$
\end{tabular}

Table S8. The FT-ICR-MS list of the featured fragments of 2,5-PyDCDs-P (positive mode)

\begin{tabular}{cccccc}
\hline \hline $\begin{array}{c}\text { Fragment Experimental } \\
\text { molecular } \\
\text { weight } \\
(\mathrm{m} / \mathrm{z})\end{array}$ & $\begin{array}{c}\text { Molecular } \\
\text { formula }\end{array}$ & $\begin{array}{c}\text { Calculated Error } \\
\text { molecular } \\
\text { weight } \\
(\mathrm{m} / \mathrm{z})\end{array}$ & $\begin{array}{c}\text { Sugm }) \\
\text { structure }\end{array}$ \\
\hline 25-PyD & 110.07180 & $\mathrm{C}_{5} \mathrm{H}_{8} \mathrm{~N}_{3}$ & 110.07127 & -4.77 & $25-\mathrm{PyD}+\mathrm{H}$ \\
\hline 25-PyD & 162.10350 & $\mathrm{C}_{9} \mathrm{H}_{12} \mathrm{~N}_{3}$ & 162.10257 & -5.69 & {$\left[(25-\mathrm{PyD})+\left(\mathrm{C}_{2} \mathrm{H}_{4}\right)_{2}-4 \mathrm{H}\right]+\mathrm{H}$} \\
\hline 25-PyD & 174.06758 & $\mathrm{C}_{9} \mathrm{H}_{8} \mathrm{~N}_{3} \mathrm{O}$ & 174.06619 & -7.99 & {$\left[(25-\mathrm{PyD})+\left(\mathrm{C}_{2} \mathrm{H}_{4}\right)+\left(\mathrm{C}_{2} \mathrm{H}_{4} \mathrm{O}\right)-8 \mathrm{H}\right]+\mathrm{H}$} \\
\hline 25-PyD & 186.10630 & $\mathrm{C}_{11} \mathrm{H}_{12} \mathrm{~N}_{3}$ & 186.10257 & -19.04 & {$\left[(25-\mathrm{PyD})+\left(\mathrm{C}_{2} \mathrm{H}_{4}\right)_{3}-8 \mathrm{H}\right]+\mathrm{H}$} \\
\hline 25-PyD & 208.14566 & $\mathrm{C}_{11} \mathrm{H}_{18} \mathrm{~N}_{3} \mathrm{O}$ & 208.14444 & -5.87 & {$\left[(25-\mathrm{PyD})+\left(\mathrm{C}_{2} \mathrm{H}_{4}\right)_{2}+\left(\mathrm{C}_{2} \mathrm{H} \mathrm{H}_{4} \mathrm{O}\right)-2 \mathrm{H}\right]+\mathrm{H}$} \\
\hline 25-PyD & 214.13524 & $\mathrm{C}_{13} \mathrm{H}_{16} \mathrm{~N}_{3}$ & 214.13387 & -6.38 & {$\left[(25-\mathrm{PyD})+\left(\mathrm{C}_{2} \mathrm{H}_{4}\right)_{4}-8 \mathrm{H}\right]+\mathrm{H}$} \\
\hline EtOH & 217.10470 & $\mathrm{C}_{10} \mathrm{H}_{17} \mathrm{O}_{5}$ & 217.10705 & 10.84 & {$\left[\left(\mathrm{C}_{2} \mathrm{H}_{4} \mathrm{O}\right)_{5}-4 \mathrm{H}\right]+\mathrm{H}$} \\
\hline 25-PyD & 226.09643 & $\mathrm{C}_{13} \mathrm{H}_{12} \mathrm{~N}_{3} \mathrm{O}$ & 226.09749 & 4.66 & {$\left[(25-\mathrm{PyD})+\left(\mathrm{C}_{2} \mathrm{H}_{4}\right)_{3}+\left(\mathrm{C}_{2} \mathrm{H}_{4} \mathrm{O}\right)-12 \mathrm{H}\right]+\mathrm{H}$} \\
\hline 25-PyD & 243.13683 & $\mathrm{C}_{12} \mathrm{H}_{13} \mathrm{~N}_{6}$ & 243.13527 & -6.43 & {$\left[(25-\mathrm{PyD})_{2}+\left(\mathrm{C}_{2} \mathrm{H}_{4}\right)-4 \mathrm{H}\right]+\mathrm{H}$} \\
\hline 25-PyD & 271.16844 & $\mathrm{C}_{14} \mathrm{H}_{19} \mathrm{~N}_{6}$ & 271.16657 & -6.91 & {$\left[(25-\mathrm{PyD})_{2}+\left(\mathrm{C}_{2} \mathrm{H}_{4}\right)_{2}-4 \mathrm{H}\right]+\mathrm{H}$} \\
\hline
\end{tabular}




\begin{tabular}{|c|c|c|c|c|c|}
\hline EtOH & 305.15951 & $\mathrm{C}_{14} \mathrm{H}_{25} \mathrm{O}_{7}$ & 305.15948 & -0.11 & {$\left[\left(\mathrm{C}_{2} \mathrm{H}_{4} \mathrm{O}\right)_{7}-4 \mathrm{H}\right]+\mathrm{H}$} \\
\hline EtOH & 349.18577 & $\mathrm{C}_{16} \mathrm{H}_{29} \mathrm{O}_{8}$ & 349.18569 & -0.22 & {$\left[\left(\mathrm{C}_{2} \mathrm{H}_{4} \mathrm{O}\right)_{8}-4 \mathrm{H}\right]+\mathrm{H}$} \\
\hline EtOH & 360.32533 & $\mathrm{C}_{24} \mathrm{H}_{42} \mathrm{NO}$ & 360.32609 & 2.11 & {$\left[\left(\mathrm{C}_{2} \mathrm{H}_{4}\right)_{11}+\left(\mathrm{C}_{2} \mathrm{H}_{4} \mathrm{O}\right)+\mathrm{NH}_{3}-10 \mathrm{H}\right]+\mathrm{H}$} \\
\hline 25-PyD & 372.17118 & $\mathrm{C}_{19} \mathrm{H}_{18} \mathrm{~N}_{9}$ & 372.16797 & -8.63 & {$\left[(25-\mathrm{PyD})_{3}+\left(\mathrm{C}_{2} \mathrm{H}_{4}\right)_{2}-16 \mathrm{H}\right]+\mathrm{H}$} \\
\hline EtOH & 374.30614 & $\mathrm{C}_{24} \mathrm{H}_{40} \mathrm{NO}_{2}$ & 360.32609 & -2.11 & {$\left[\left(\mathrm{C}_{2} \mathrm{H}_{4}\right)_{10}+\left(\mathrm{C}_{2} \mathrm{H}_{4} \mathrm{O}\right)_{2}+\mathrm{NH}_{3}-12 \mathrm{H}\right]+\mathrm{H}$} \\
\hline EtOH & 388.35846 & $\mathrm{C}_{26} \mathrm{H}_{46} \mathrm{NO}$ & 388.35739 & -2.75 & {$\left[\left(\mathrm{C}_{2} \mathrm{H}_{4}\right)_{12}+\left(\mathrm{C}_{2} \mathrm{H}_{4} \mathrm{O}\right)+\mathrm{NH}_{3}-10 \mathrm{H}\right]+\mathrm{H}$} \\
\hline ЕtOH & 392.31696 & $\mathrm{C}_{24} \mathrm{H}_{42} \mathrm{NO}_{3}$ & 392.31592 & -2.64 & {$\left[\left(\mathrm{C}_{2} \mathrm{H}_{4}\right)_{9}+\left(\mathrm{C}_{2} \mathrm{H}_{4} \mathrm{O}\right)_{3}+\mathrm{NH}_{3}-8 \mathrm{H}\right]+\mathrm{H}$} \\
\hline EtOH & 393.20963 & $\mathrm{C}_{18} \mathrm{H}_{33} \mathrm{O}_{9}$ & 393.21191 & 5.78 & {$\left[\left(\mathrm{C}_{2} \mathrm{H}_{4} \mathrm{O}\right)_{9}-4 \mathrm{H}\right]+\mathrm{H}$} \\
\hline 25-PyD & 400.20270 & $\mathrm{C}_{21} \mathrm{H}_{22} \mathrm{~N}_{9}$ & 400.19927 & -0.53 & {$\left[(25-\mathrm{PyD})_{3}+\left(\mathrm{C}_{2} \mathrm{H}_{4}\right)_{3}-12 \mathrm{H}\right]+\mathrm{H}$} \\
\hline EtOH & 437.23921 & $\mathrm{C}_{20} \mathrm{H}_{37} \mathrm{O}_{10}$ & 437.23812 & -2.48 & {$\left[\left(\mathrm{C}_{2} \mathrm{H}_{4} \mathrm{O}\right)_{10^{-}}-4 \mathrm{H}\right]+\mathrm{H}$} \\
\hline EtOH & 481.26472 & $\mathrm{C}_{22} \mathrm{H}_{41} \mathrm{O}_{11}$ & 481.26434 & -0.79 & {$\left[\left(\mathrm{C}_{2} \mathrm{H}_{4} \mathrm{O}\right)_{11}-4 \mathrm{H}\right]+\mathrm{H}$} \\
\hline EtOH & 525.29327 & $\mathrm{C}_{24} \mathrm{H}_{45} \mathrm{O}_{12}$ & 525.29055 & -5.18 & {$\left[\left(\mathrm{C}_{2} \mathrm{H}_{4} \mathrm{O}\right)_{12}-4 \mathrm{H}\right]+\mathrm{H}$} \\
\hline EtOH & 569.32026 & $\mathrm{C}_{26} \mathrm{H}_{49} \mathrm{O}_{13}$ & 569.31677 & -6.13 & {$\left[\left(\mathrm{C}_{2} \mathrm{H}_{4} \mathrm{O}\right)_{13}-4 \mathrm{H}\right]+\mathrm{H}$} \\
\hline EtOH & 613.34761 & $\mathrm{C}_{28} \mathrm{H}_{53} \mathrm{O}_{14}$ & 613.34298 & -7.54 & {$\left[\left(\mathrm{C}_{2} \mathrm{H}_{4} \mathrm{O}\right)_{14}-4 \mathrm{H}\right]+\mathrm{H}$} \\
\hline EtOH & 657.37336 & $\mathrm{C}_{30} \mathrm{H}_{57} \mathrm{O}_{15}$ & 657.36920 & -6.34 & {$\left[\left(\mathrm{C}_{2} \mathrm{H}_{4} \mathrm{O}\right)_{15}-4 \mathrm{H}\right]+\mathrm{H}$} \\
\hline EtOH & 745.42716 & $\mathrm{C}_{34} \mathrm{H}_{65} \mathrm{O}_{17}$ & 745.42163 & -7.42 & {$\left[\left(\mathrm{C}_{2} \mathrm{H}_{4} \mathrm{O}\right)_{17}-4 \mathrm{H}\right]+\mathrm{H}$} \\
\hline EtOH & 789.45654 & $\mathrm{C}_{36} \mathrm{H}_{69} \mathrm{O}_{18}$ & 789.44784 & -11.02 & {$\left[\left(\mathrm{C}_{2} \mathrm{H}_{4} \mathrm{O}\right)_{18}-4 \mathrm{H}\right]+\mathrm{H}$} \\
\hline EtOH & 833.47625 & $\mathrm{C}_{38} \mathrm{H}_{73} \mathrm{O}_{19}$ & 833.47406 & -2.63 & {$\left[\left(\mathrm{C}_{2} \mathrm{H}_{4} \mathrm{O}\right)_{19^{-}}-4 \mathrm{H}\right]+\mathrm{H}$} \\
\hline
\end{tabular}

Table S9. The FT-ICR-MS list of the featured fragments of $m$-PDCDs-P (positive mode)

\begin{tabular}{cccccc}
\hline \hline $\begin{array}{c}\text { Fragment } \\
\text { Species }\end{array}$ & $\begin{array}{c}\text { Experimental } \\
\text { molecular } \\
\text { weight } \\
(\mathrm{m} / \mathrm{z})\end{array}$ & $\begin{array}{c}\text { Molecular } \\
\text { formula }\end{array}$ & $\begin{array}{c}\text { Calculated } \\
\text { molecular } \\
\text { weight } \\
(\mathrm{m} / \mathrm{z})\end{array}$ & $\begin{array}{c}\text { Error } \\
(\mathrm{ppm})\end{array}$ & $\begin{array}{c}\text { Suggested } \\
\text { structure }\end{array}$ \\
\hline $\boldsymbol{m}$-PD & 159.09288 & $\mathrm{C}_{10} \mathrm{H}_{8} \mathrm{~N}_{2}$ & 159.07127 & 4.77 & $m$-PD+H \\
\hline $\boldsymbol{m}$-PD & 175.08740 & $\mathrm{C}_{10} \mathrm{H}_{11} \mathrm{~N}_{2} \mathrm{O}$ & 175.08659 & -4.63 & {$\left[\left(\mathrm{C}_{2} \mathrm{H}_{4} \mathrm{O}\right)_{4}-2 \mathrm{H}\right]+\mathrm{H}$} \\
\hline $\boldsymbol{m}$-PD & 187.12399 & $\mathrm{C}_{12} \mathrm{H}_{15} \mathrm{~N}_{2}$ & 187.12297 & -5.44 & {$\left[(m-\mathrm{PD})+\left(\mathrm{C}_{2} \mathrm{H}_{4}\right)_{3}-6 \mathrm{H}\right]+\mathrm{H}$} \\
\hline $\boldsymbol{m}$-PD & 265.14421 & $\mathrm{C}_{16} \mathrm{H}_{17} \mathrm{~N}_{4}$ & 265.14477 & 2.14 & {$\left[(m-\mathrm{PD})_{2}+\left(\mathrm{C}_{2} \mathrm{H}_{4}\right)_{2}-8 \mathrm{H}\right]+\mathrm{H}$} \\
\hline $\boldsymbol{m}$-PD & 279.12603 & $\mathrm{C}_{16} \mathrm{H}_{15} \mathrm{~N}_{4} \mathrm{O}$ & 265.14477 & 2.14 & {$\left[(m-\mathrm{PD})_{2}+\left(\mathrm{C}_{2} \mathrm{H}_{4}\right)+\left(\mathrm{C}_{2} \mathrm{H}_{4} \mathrm{O}\right)-10 \mathrm{H}\right]+\mathrm{H}$} \\
\hline $\boldsymbol{m}$-PD & 293.17805 & $\mathrm{C}_{18} \mathrm{H}_{21} \mathrm{~N}_{4}$ & 293.17607 & -6.75 & {$\left[(m-\mathrm{PD})_{2}+\left(\mathrm{C}_{2} \mathrm{H}_{4}\right)_{3}-8 \mathrm{H}\right]+\mathrm{H}$} \\
\hline EtOH & 305.15953 & $\mathrm{C}_{14} \mathrm{H}_{25} \mathrm{O}_{7}$ & 305.15948 & -0.17 & {$\left[\left(\mathrm{C}_{2} \mathrm{H}_{4} \mathrm{O}\right)_{7}-4 \mathrm{H}\right]+\mathrm{H}$}
\end{tabular}




\begin{tabular}{|c|c|c|c|c|c|}
\hline$m-P D$ & 346.16941 & $\mathrm{C}_{20} \mathrm{H}_{20} \mathrm{~N}_{5} \mathrm{O}$ & 346.17211 & 7.81 & {$\left[(m-\mathrm{PD})_{3}-\mathrm{NH}+\left(\mathrm{C}_{2} \mathrm{H}_{4} \mathrm{O}\right)-12 \mathrm{H}\right]+\mathrm{H}$} \\
\hline EtOH & 349.18575 & $\mathrm{C}_{16} \mathrm{H}_{29} \mathrm{O}_{8}$ & 349.18569 & -0.17 & {$\left[\left(\mathrm{C}_{2} \mathrm{H}_{4} \mathrm{O}\right)_{8}-4 \mathrm{H}\right]+\mathrm{H}$} \\
\hline EtOH & 360.32533 & $\mathrm{C}_{24} \mathrm{H}_{42} \mathrm{NO}$ & 360.32609 & 2.11 & {$\left[\left(\mathrm{C}_{2} \mathrm{H}_{4}\right)_{11}+\left(\mathrm{C}_{2} \mathrm{H}_{4} \mathrm{O}\right)+\mathrm{NH}_{3}-10 \mathrm{H}\right]+\mathrm{H}$} \\
\hline$m-P D$ & 370.16970 & $\mathrm{C}_{22} \mathrm{H}_{20} \mathrm{~N}_{5} \mathrm{O}$ & 370.16624 & -9.37 & {$\left[(m-\mathrm{PD})_{3}-\mathrm{NH}+\left(\mathrm{C}_{2} \mathrm{H}_{4} \mathrm{O}\right)-16 \mathrm{H}\right]+\mathrm{H}$} \\
\hline EtOH & 374.30628 & $\mathrm{C}_{24} \mathrm{H}_{40} \mathrm{NO}_{2}$ & 374.30536 & -2.11 & {$\left[\left(\mathrm{C}_{2} \mathrm{H}_{4}\right)_{10}+\left(\mathrm{C}_{2} \mathrm{H}_{4} \mathrm{O}\right)_{2}+\mathrm{NH}_{3}-12 \mathrm{H}\right]+\mathrm{H}$} \\
\hline EtOH & 376.30113 & $\mathrm{C}_{20} \mathrm{H}_{42} \mathrm{NO}_{5}$ & 376.30575 & 12.28 & {$\left[\left(\mathrm{C}_{2} \mathrm{H}_{4}\right)_{5}+\left(\mathrm{C}_{2} \mathrm{H}_{4} \mathrm{O}\right)_{5}+\mathrm{NH}_{3}-2 \mathrm{H}\right]+\mathrm{H}$} \\
\hline EtOH & 388.35865 & $\mathrm{C}_{26} \mathrm{H}_{46} \mathrm{NO}$ & 388.35739 & -3.24 & {$\left[\left(\mathrm{C}_{2} \mathrm{H}_{4}\right)_{12}+\left(\mathrm{C}_{2} \mathrm{H}_{4} \mathrm{O}\right)+\mathrm{NH}_{3}-10 \mathrm{H}\right]+\mathrm{H}$} \\
\hline EtOH & 392.31721 & $\mathrm{C}_{24} \mathrm{H}_{42} \mathrm{NO}_{3}$ & 392.31592 & -3.28 & {$\left[\left(\mathrm{C}_{2} \mathrm{H}_{4}\right)_{9}+\left(\mathrm{C}_{2} \mathrm{H}_{4} \mathrm{O}\right)_{3}+\mathrm{NH}_{3}-10 \mathrm{H}\right]+\mathrm{H}$} \\
\hline EtOH & 393.21351 & $\mathrm{C}_{18} \mathrm{H}_{33} \mathrm{O}_{9}$ & 393.21191 & -4.07 & {$\left[\left(\mathrm{C}_{2} \mathrm{H}_{4} \mathrm{O}\right)_{9}-4 \mathrm{H}\right]+\mathrm{H}$} \\
\hline$m-\mathbf{P D}$ & 399.23259 & $\mathrm{C}_{24} \mathrm{H}_{27} \mathrm{~N}_{6}$ & 399.22917 & -8.57 & {$\left[(m-\mathrm{PD})_{3}+\left(\mathrm{C}_{2} \mathrm{H}_{4}\right)_{3}-10 \mathrm{H}\right]+\mathrm{H}$} \\
\hline EtOH & 437.24006 & $\mathrm{C}_{20} \mathrm{H}_{37} \mathrm{O}_{10}$ & 437.23812 & -4.44 & {$\left[\left(\mathrm{C}_{2} \mathrm{H}_{4} \mathrm{O}\right)_{10^{-}}-4 \mathrm{H}\right]+\mathrm{H}$} \\
\hline EtOH & 481.26631 & $\mathrm{C}_{22} \mathrm{H}_{41} \mathrm{O}_{11}$ & 481.26434 & -4.10 & {$\left[\left(\mathrm{C}_{2} \mathrm{H}_{4} \mathrm{O}\right)_{11}-4 \mathrm{H}\right]+\mathrm{H}$} \\
\hline EtOH & 525.29378 & $\mathrm{C}_{24} \mathrm{H}_{45} \mathrm{O}_{12}$ & 525.29055 & -6.14 & {$\left[\left(\mathrm{C}_{2} \mathrm{H}_{4} \mathrm{O}\right)_{12}-4 \mathrm{H}\right]+\mathrm{H}$} \\
\hline EtOH & 569.31960 & $\mathrm{C}_{26} \mathrm{H}_{49} \mathrm{O}_{13}$ & 569.31677 & -4.98 & {$\left[\left(\mathrm{C}_{2} \mathrm{H}_{4} \mathrm{O}\right)_{13}-4 \mathrm{H}\right]+\mathrm{H}$} \\
\hline EtOH & 613.34807 & $\mathrm{C}_{28} \mathrm{H}_{53} \mathrm{O}_{14}$ & 613.34298 & -8.30 & {$\left[\left(\mathrm{C}_{2} \mathrm{H}_{4} \mathrm{O}\right)_{14-4}-4 \mathrm{H}\right]+\mathrm{H}$} \\
\hline EtOH & 657.37424 & $\mathrm{C}_{30} \mathrm{H}_{57} \mathrm{O}_{15}$ & 657.36920 & -7.68 & {$\left[\left(\mathrm{C}_{2} \mathrm{H}_{4} \mathrm{O}\right)_{15}-4 \mathrm{H}\right]+\mathrm{H}$} \\
\hline EtOH & 745.42956 & $\mathrm{C}_{34} \mathrm{H}_{65} \mathrm{O}_{17}$ & 745.42163 & -10.64 & {$\left[\left(\mathrm{C}_{2} \mathrm{H}_{4} \mathrm{O}\right)_{17}-4 \mathrm{H}\right]+\mathrm{H}$} \\
\hline EtOH & 789.44679 & $\mathrm{C}_{36} \mathrm{H}_{69} \mathrm{O}_{18}$ & 789.44784 & 1.33 & {$\left[\left(\mathrm{C}_{2} \mathrm{H}_{4} \mathrm{O}\right)_{18}-4 \mathrm{H}\right]+\mathrm{H}$} \\
\hline
\end{tabular}

Table S10. The FT-ICR-MS list of the featured fragments of $o$-PDCDs-P(positive mode)

\begin{tabular}{|c|c|c|c|c|c|}
\hline $\begin{array}{c}\text { Fragment } \\
\text { Species }\end{array}$ & $\begin{array}{c}\text { Experimental } \\
\text { nolecularwei } \\
\mathrm{ht} \\
(\mathrm{m} / \mathrm{z})\end{array}$ & $\begin{array}{c}\begin{array}{c}\text { Molecularfo } \\
\text { mula }\end{array} \\
\text { and }\end{array}$ & $\begin{array}{c}\text { Calculated } \\
\text { molecular } \\
\text { weight } \\
(\mathrm{m} / \mathrm{z})\end{array}$ & $\begin{array}{l}\text { Error } \\
(\mathrm{ppm})\end{array}$ & $\begin{array}{l}\text { Suggested } \\
\text { structure }\end{array}$ \\
\hline$o$-PD & 133.07665 & $\mathrm{C}_{8} \mathrm{H}_{9} \mathrm{~N}_{2}$ & 133.07602 & -4.68 & {$\left[(o-\mathrm{PD})+\left(\mathrm{C}_{2} \mathrm{H}_{4}\right)-4 \mathrm{H}\right]+\mathrm{H}$} \\
\hline$o$-PD & 155.05885 & $\mathrm{C}_{10} \mathrm{H}_{7} \mathrm{~N}_{2}$ & 155.06037 & 9.86 & {$\left[o-\mathrm{PD}+\left(\mathrm{C}_{2} \mathrm{H}_{4}\right)_{2}-8 \mathrm{H}\right]+\mathrm{H}$} \\
\hline$o$-PD & 161.10814 & $\mathrm{C}_{10} \mathrm{H}_{13} \mathrm{~N}_{2}$ & 161.10732 & -5.06 & {$\left[o-\mathrm{PD}+\left(\mathrm{C}_{2} \mathrm{H}_{4}\right)_{2}-2 \mathrm{H}\right]+\mathrm{H}$} \\
\hline$o$-PD & 175.09898 & $\mathrm{C}_{10} \mathrm{H}_{11} \mathrm{~N}_{2} \mathrm{O}$ & 175.08659 & -4.63 & {$\left[\left(\mathrm{C}_{2} \mathrm{H}_{4} \mathrm{O}\right)_{4}-2 \mathrm{H}\right]+\mathrm{H}$} \\
\hline$o$-PD & 183.09060 & $\mathrm{C}_{12} \mathrm{H}_{11} \mathrm{~N}_{2}$ & 183.09167 & 5.88 & {$\left[(o-\mathrm{PD})+\left(\mathrm{C}_{6} \mathrm{H}_{6}\right)-4 \mathrm{H}\right]+\mathrm{H}$} \\
\hline
\end{tabular}




\begin{tabular}{|c|c|c|c|c|c|}
\hline$o-P D$ & 187.15367 & $\mathrm{C}_{8} \mathrm{H}_{19} \mathrm{~N}_{4} \mathrm{O}$ & 187.15534 & 8.91 & {$\left[(o-\mathrm{PD})+\left(\mathrm{C}_{2} \mathrm{H}_{4} \mathrm{O}\right)+2 \mathrm{NH}_{3}\right]+\mathrm{H}$} \\
\hline$o$-PD & 205.13470 & $\mathrm{C}_{12} \mathrm{H}_{17} \mathrm{~N}_{2} \mathrm{O}$ & 205.13354 & -5.63 & {$\left[(o-\mathrm{PD})+\left(\mathrm{C}_{2} \mathrm{H}_{4}\right)_{2}+\left(\mathrm{C}_{2} \mathrm{H}_{4} \mathrm{O}\right)-4 \mathrm{H}\right]+\mathrm{H}$} \\
\hline$o$-PD & 213.06515 & $\mathrm{C}_{12} \mathrm{H}_{9} \mathrm{~N}_{2} \mathrm{O}_{2}$ & 213.06317 & -9.28 & {$\left[(o-\mathrm{PD})+\left(\mathrm{C}_{6} \mathrm{H}_{6} \mathrm{O}_{2}\right)-6 \mathrm{H}\right]+\mathrm{H}$} \\
\hline$o$-PD & 227.11681 & $\mathrm{C}_{14} \mathrm{H}_{15} \mathrm{~N}_{2} \mathrm{O}$ & 227.11789 & 4.77 & {$\left[(o-\mathrm{PD})+\left(\mathrm{C}_{2} \mathrm{H}_{4}\right)_{3}+\left(\mathrm{C}_{2} \mathrm{H}_{4} \mathrm{O}\right)-10 \mathrm{H}\right]+\mathrm{H}$} \\
\hline$o-P D$ & 241.09626 & $\mathrm{C}_{14} \mathrm{H}_{13} \mathrm{~N}_{2} \mathrm{O}_{2}$ & 241.09715 & 3.70 & {$\left[(o-\mathrm{PD})+\left(\mathrm{C}_{2} \mathrm{H}_{4}\right)_{2}+\left(\mathrm{C}_{2} \mathrm{H}_{4} \mathrm{O}\right)_{2}-12 \mathrm{H}\right]+\mathrm{H}$} \\
\hline$o-P D$ & 261.13189 & $\mathrm{C}_{12} \mathrm{H}_{21} \mathrm{O}_{6}$ & 261.13326 & 5.26 & {$\left[\left(\mathrm{C}_{2} \mathrm{H}_{4} \mathrm{O}\right)_{6}-2 \mathrm{H}\right]+\mathrm{H}$} \\
\hline$o-P D$ & 289.09687 & $\mathrm{C}_{18} \mathrm{H}_{13} \mathrm{~N}_{2} \mathrm{O}_{2}$ & 289.09715 & 1.00 & {$\left[(o-\mathrm{PD})+\left(\mathrm{C}_{6} \mathrm{H}_{6}\right)+\left(\mathrm{C}_{6} \mathrm{H}_{6} \mathrm{O}_{2}\right)-8 \mathrm{H}\right]+\mathrm{H}$} \\
\hline EtOH & 305.15952 & $\mathrm{C}_{14} \mathrm{H}_{25} \mathrm{O}_{7}$ & 305.15948 & -0.13 & {$\left[\left(\mathrm{C}_{2} \mathrm{H}_{4} \mathrm{O}\right)_{7}-4 \mathrm{H}\right]+\mathrm{H}$} \\
\hline$o$-PD & 317.05304 & $\mathrm{C}_{18} \mathrm{H}_{9} \mathrm{~N}_{2} \mathrm{O}_{4}$ & 317.05568 & 8.34 & {$\left[(o-\mathrm{PD})+\left(\mathrm{C}_{6} \mathrm{H}_{6} \mathrm{O}_{2}\right)_{2}-12 \mathrm{H}\right]+\mathrm{H}$} \\
\hline$o$-PD & 323.10248 & $\mathrm{C}_{18} \mathrm{H}_{15} \mathrm{~N}_{2} \mathrm{O}_{4}$ & 323.10263 & 0.47 & {$\left[(o-\mathrm{PD})+\left(\mathrm{C}_{6} \mathrm{H}_{6} \mathrm{O}_{2}\right)_{2}-6 \mathrm{H}\right]+\mathrm{H}$} \\
\hline EtOH & 349.18578 & $\mathrm{C}_{16} \mathrm{H}_{29} \mathrm{O}_{8}$ & 349.18569 & -0.26 & {$\left[\left(\mathrm{C}_{2} \mathrm{H}_{4} \mathrm{O}\right)_{8}-4 \mathrm{H}\right]+\mathrm{H}$} \\
\hline EtOH & 360.32533 & $\mathrm{C}_{24} \mathrm{H}_{42} \mathrm{NO}$ & 360.32609 & 2.11 & {$\left[\left(\mathrm{C}_{2} \mathrm{H}_{4}\right)_{11}+\left(\mathrm{C}_{2} \mathrm{H}_{4} \mathrm{O}\right)+\mathrm{NH}_{3}-10 \mathrm{H}\right]+\mathrm{H}$} \\
\hline$o-P D$ & 370.16970 & $\mathrm{C}_{22} \mathrm{H}_{20} \mathrm{~N}_{5} \mathrm{O}$ & 370.16624 & -9.37 & {$\left[(o-\mathrm{PD})_{3}-\mathrm{NH}+\left(\mathrm{C}_{2} \mathrm{H}_{4} \mathrm{O}\right)-16 \mathrm{H}\right]+\mathrm{H}$} \\
\hline EtOH & 374.30587 & $\mathrm{C}_{24} \mathrm{H}_{40} \mathrm{NO}_{2}$ & 374.30536 & -1.36 & {$\left[\left(\mathrm{C}_{2} \mathrm{H}_{4}\right)_{10}+\left(\mathrm{C}_{2} \mathrm{H}_{4} \mathrm{O}\right)_{2}+\mathrm{NH}_{3}-12 \mathrm{H}\right]+\mathrm{H}$} \\
\hline EtOH & 376.30063 & $\mathrm{C}_{20} \mathrm{H}_{42} \mathrm{NO}_{5}$ & 376.30575 & 13.60 & {$\left[\left(\mathrm{C}_{2} \mathrm{H}_{4}\right)_{5}+\left(\mathrm{C}_{2} \mathrm{H}_{4} \mathrm{O}\right)_{5}+\mathrm{NH}_{3}-2 \mathrm{H}\right]+\mathrm{H}$} \\
\hline EtOH & 388.35814 & $\mathrm{C}_{26} \mathrm{H}_{46} \mathrm{NO}$ & 388.35739 & -1.94 & {$\left[\left(\mathrm{C}_{2} \mathrm{H}_{4}\right)_{12}+\left(\mathrm{C}_{2} \mathrm{H}_{4} \mathrm{O}\right)+\mathrm{NH}_{3}-10 \mathrm{H}\right]+\mathrm{H}$} \\
\hline EtOH & 392.31673 & $\mathrm{C}_{24} \mathrm{H}_{42} \mathrm{NO}_{3}$ & 392.31592 & -2.05 & {$\left[\left(\mathrm{C}_{2} \mathrm{H}_{4}\right)_{9}+\left(\mathrm{C}_{2} \mathrm{H}_{4} \mathrm{O}\right)_{3}+\mathrm{NH}_{3}-10 \mathrm{H}\right]+\mathrm{H}$} \\
\hline EtOH & 393.21276 & $\mathrm{C}_{18} \mathrm{H}_{33} \mathrm{O}_{9}$ & 393.21191 & -2.15 & {$\left[\left(\mathrm{C}_{2} \mathrm{H}_{4} \mathrm{O}\right)_{9}-4 \mathrm{H}\right]+\mathrm{H}$} \\
\hline EtOH & 437.23942 & $\mathrm{C}_{20} \mathrm{H}_{37} \mathrm{O}_{10}$ & 437.23812 & -2.96 & {$\left[\left(\mathrm{C}_{2} \mathrm{H}_{4} \mathrm{O}\right)_{10^{-}}-4 \mathrm{H}\right]+\mathrm{H}$} \\
\hline EtOH & 481.26444 & $\mathrm{C}_{22} \mathrm{H}_{41} \mathrm{O}_{11}$ & 481.26434 & -0.21 & {$\left[\left(\mathrm{C}_{2} \mathrm{H}_{4} \mathrm{O}\right)_{11}-4 \mathrm{H}\right]+\mathrm{H}$} \\
\hline EtOH & 525.29277 & $\mathrm{C}_{24} \mathrm{H}_{45} \mathrm{O}_{12}$ & 525.29055 & -4.22 & {$\left[\left(\mathrm{C}_{2} \mathrm{H}_{4} \mathrm{O}\right)_{12}-4 \mathrm{H}\right]+\mathrm{H}$} \\
\hline EtOH & 569.31954 & $\mathrm{C}_{26} \mathrm{H}_{49} \mathrm{O}_{13}$ & 569.31677 & -4.87 & {$\left[\left(\mathrm{C}_{2} \mathrm{H}_{4} \mathrm{O}\right)_{13}-4 \mathrm{H}\right]+\mathrm{H}$} \\
\hline EtOH & 613.34661 & $\mathrm{C}_{28} \mathrm{H}_{53} \mathrm{O}_{14}$ & 613.34298 & -5.91 & {$\left[\left(\mathrm{C}_{2} \mathrm{H}_{4} \mathrm{O}\right)_{14}-4 \mathrm{H}\right]+\mathrm{H}$} \\
\hline EtOH & 657.37266 & $\mathrm{C}_{30} \mathrm{H}_{57} \mathrm{O}_{15}$ & 657.36920 & -5.27 & {$\left[\left(\mathrm{C}_{2} \mathrm{H}_{4} \mathrm{O}\right)_{15}-4 \mathrm{H}\right]+\mathrm{H}$} \\
\hline EtOH & 745.42597 & $\mathrm{C}_{34} \mathrm{H}_{65} \mathrm{O}_{17}$ & 745.42163 & -5.83 & {$\left[\left(\mathrm{C}_{2} \mathrm{H}_{4} \mathrm{O}\right)_{17}-4 \mathrm{H}\right]+\mathrm{H}$} \\
\hline EtOH & 789.45323 & $\mathrm{C}_{36} \mathrm{H}_{69} \mathrm{O}_{18}$ & 789.44784 & -6.83 & {$\left[\left(\mathrm{C}_{2} \mathrm{H}_{4} \mathrm{O}\right)_{18}-4 \mathrm{H}\right]+\mathrm{H}$} \\
\hline EtOH & 833.47833 & $\mathrm{C}_{38} \mathrm{H}_{73} \mathrm{O}_{19}$ & 833.47406 & -5.13 & {$\left[\left(\mathrm{C}_{2} \mathrm{H}_{4} \mathrm{O}\right)_{19}-4 \mathrm{H}\right]+\mathrm{H}$} \\
\hline EtOH & 877.50222 & $\mathrm{C}_{40} \mathrm{H}_{77} \mathrm{O}_{20}$ & 877.50027 & -2.23 & {$\left[\left(\mathrm{C}_{2} \mathrm{H}_{4} \mathrm{O}\right)_{20}-4 \mathrm{H}\right]+\mathrm{H}$} \\
\hline
\end{tabular}




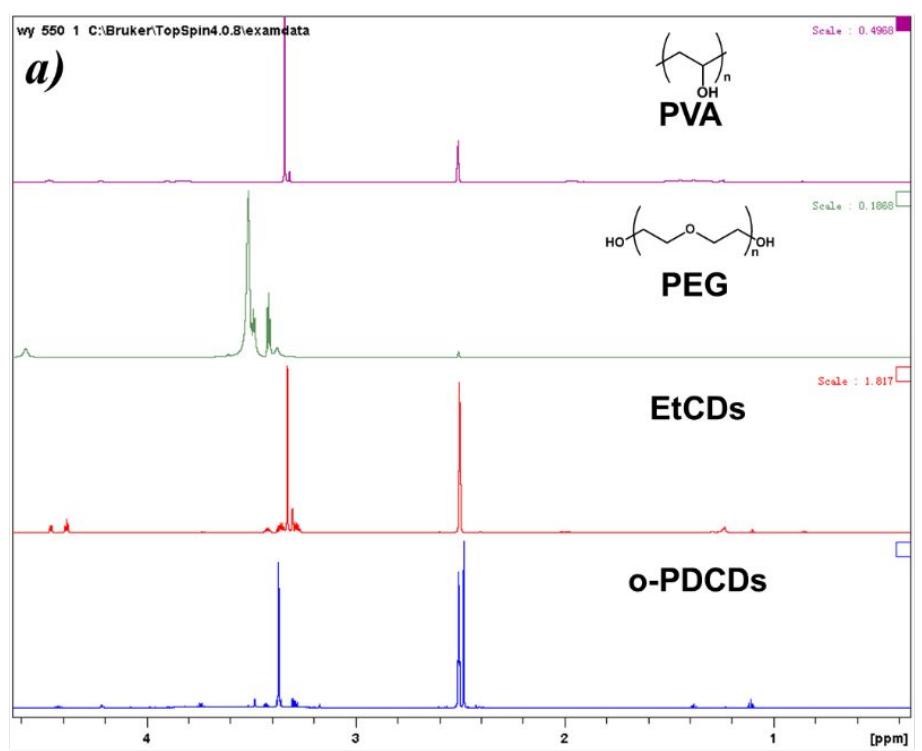

Chemical shift $\delta(p p m)$

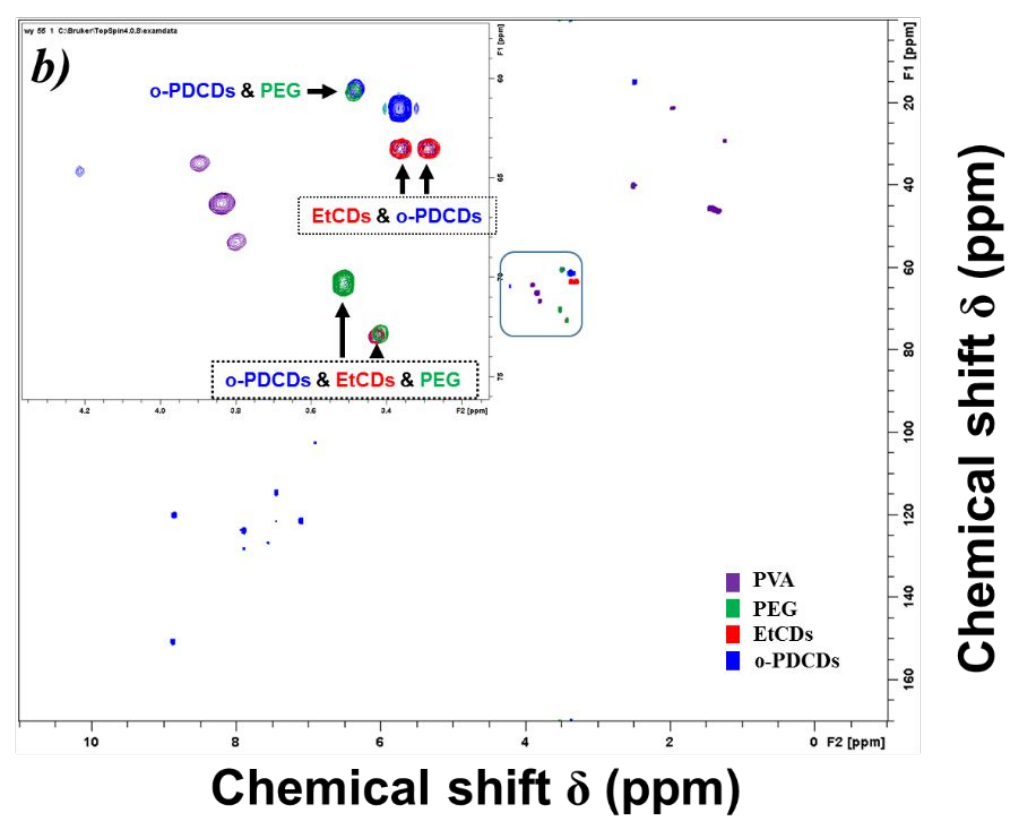

Figure S1. a) ${ }^{1} \mathrm{H}$ NMR and b) HSQC comparison of PVA, PEG, EtCDs and $o$-PDCDs. The black arrow pointed out the overlapping signals and their ascription. 


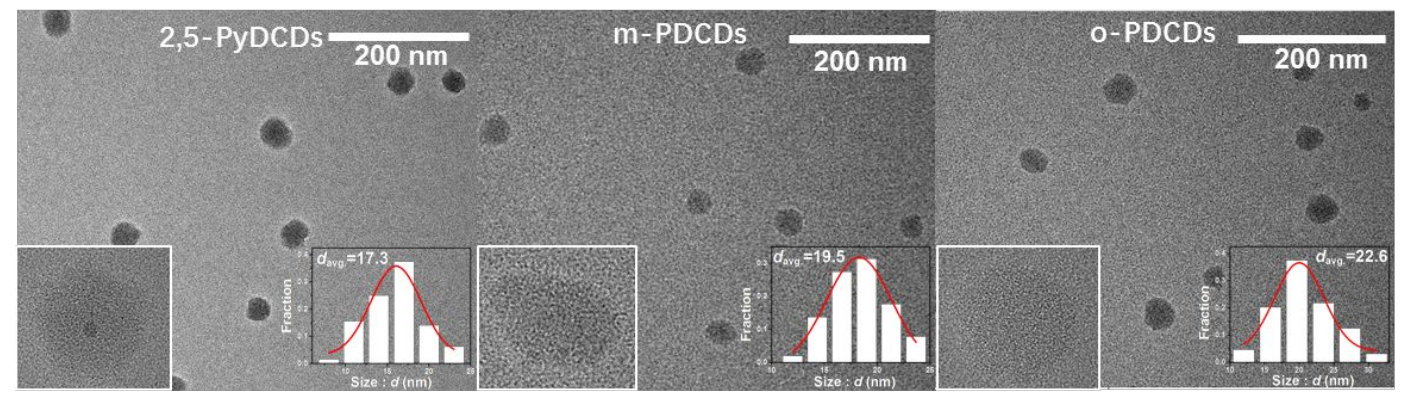

Figure S2. The TEM images of 2,5-PyDCDs, $m$-PDCD and $o$-PDCDs, the insets showed particle size distribution and HR-TEM of single particle of three CDs. 


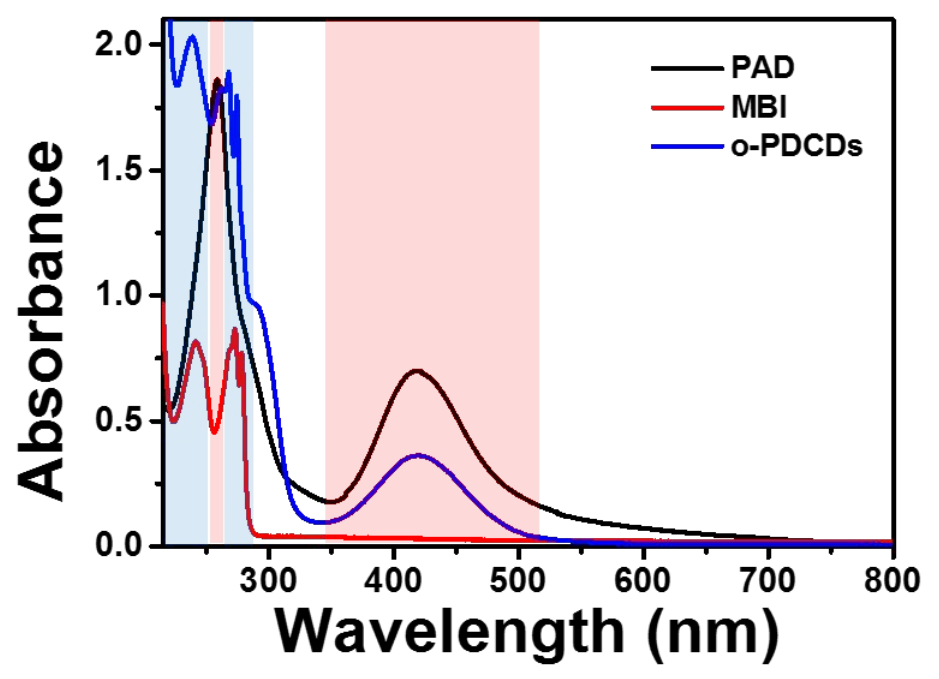

Figure S3. The absorption spectrum comparison of PAD $(0.05 \mathrm{mg} / \mathrm{mL})$, MBI $(0.05$ $\mathrm{mg} / \mathrm{ml})$ and $o$-PDCDs $(0.175 \mathrm{mg} / \mathrm{mL})$ in aqueous solution. The red area showed the band overlapping between PAD and $o$-PDCDs, and the blue area indicated the band overlapping between $\mathrm{MBI}$ and $o$-PDCDs. 


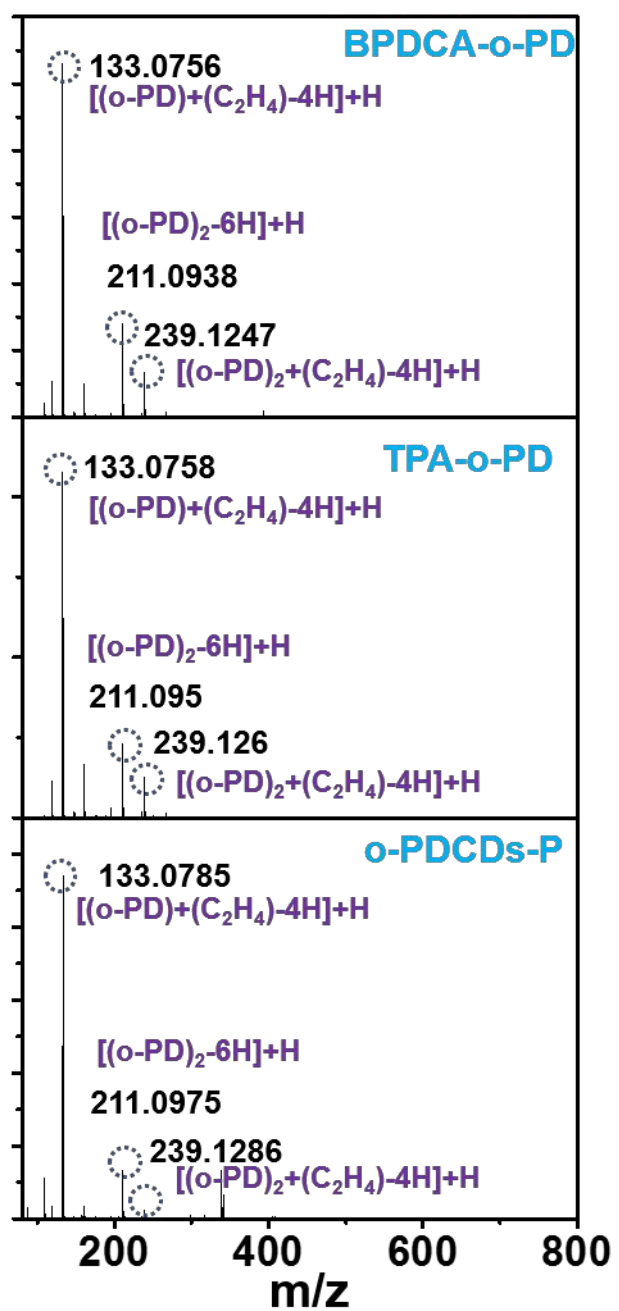

Figure S4. The UPLC-MS spectrum comparison of BPDCA- $o-\mathrm{PD}$, TPA- $o$-PD and $o$ PDCDs-P. 


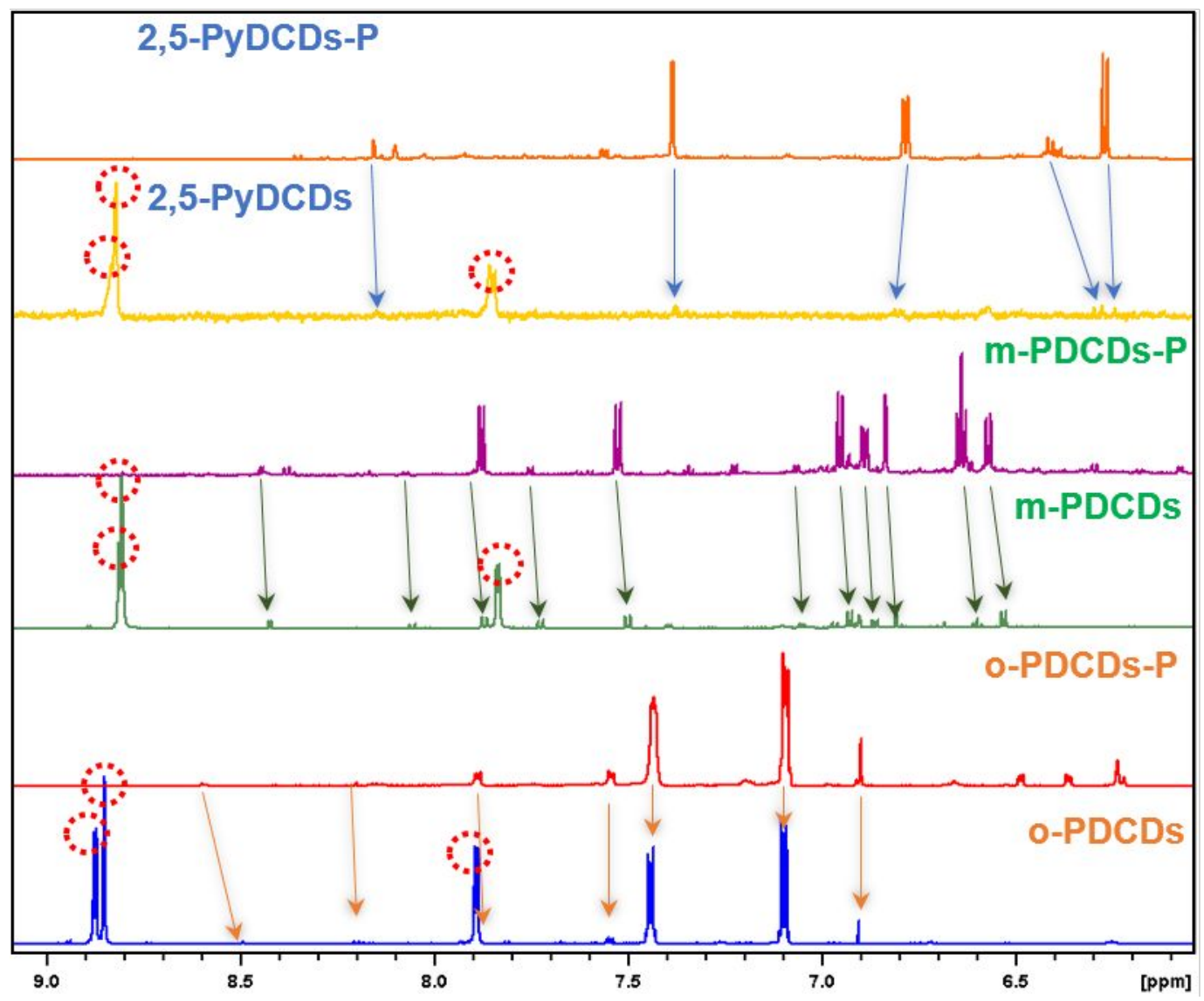

Figure S5. The a) ${ }^{1} \mathrm{H}$ NMR comparison of 2,5-PyDCDs-P, 2,5-PyDCDs, $m$-PDCDs-P, $m$-PDCDs $o$-PDCDs-P, and $o$-PDCDs. Arrow illustrated the corresponding PD/PyDrelated protons in PDCDs/PyDCDs and PDCDs-P/PyDCDs-P, read dash circles lined the protons belonging to BPyDCA. 


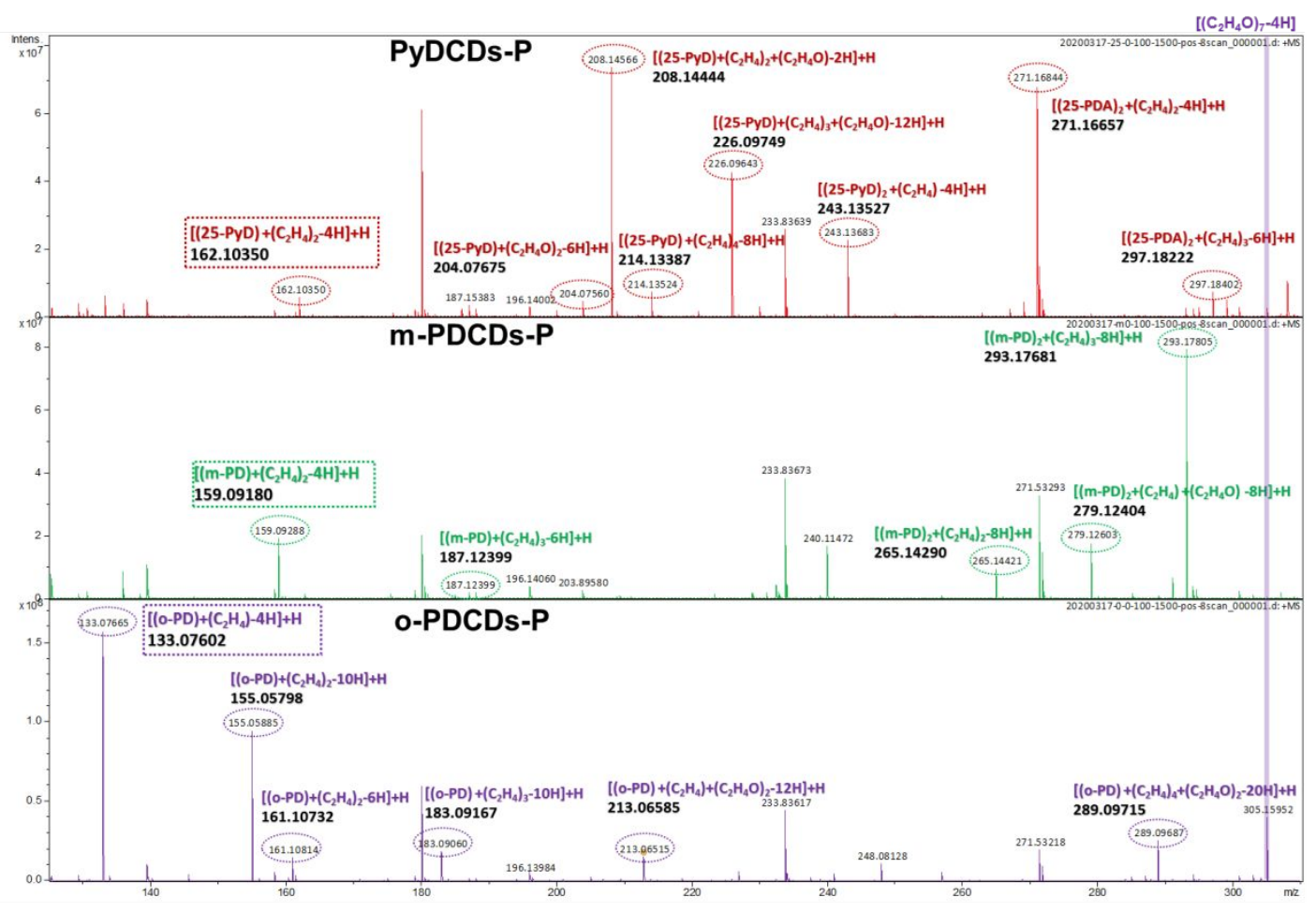

Figure S6. FT-ICR-MS of 2,5-PyDCDs-P, $m$-PDCDs-P and $o$-PDCDs-P. 


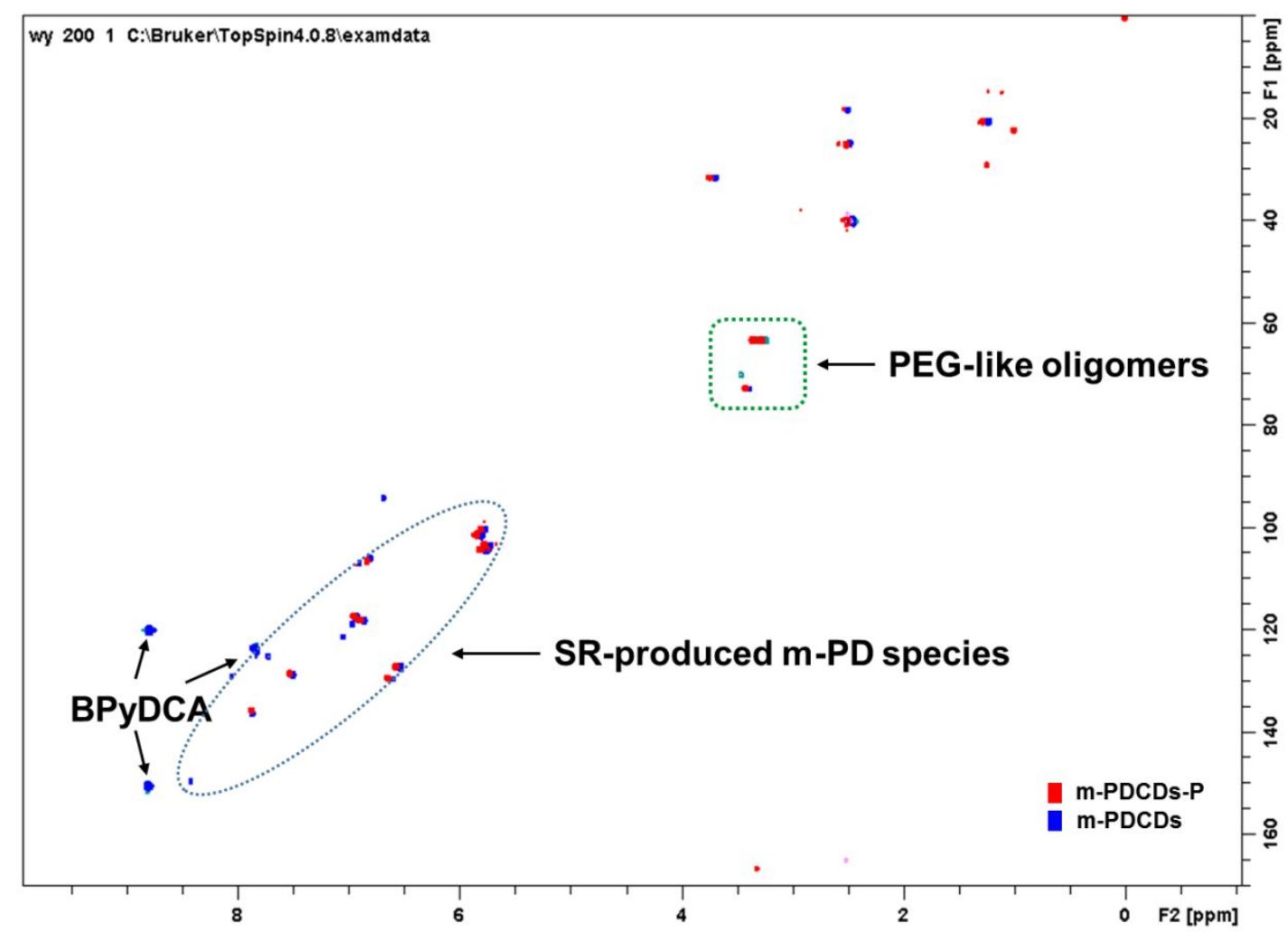

Figure S7. The HSQC comparison of $m$-PDCDs and $m$-PDCDs-P. 


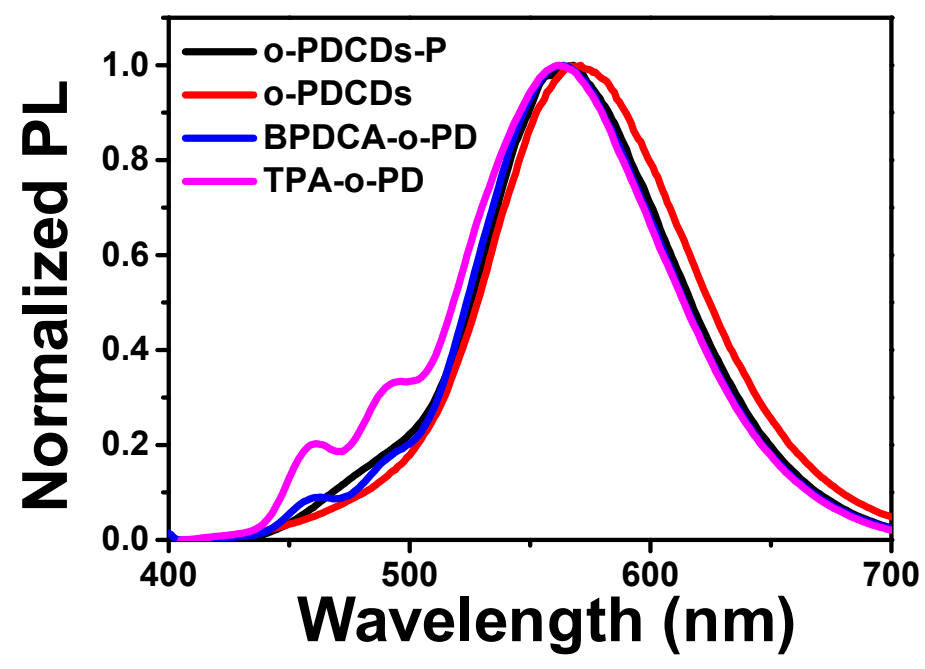

Figure S8. The emission spectrum comparison of $o$-PDCDs-P, $o$-PDCDs, BPDCA-oPD, TPA-o-PD. 


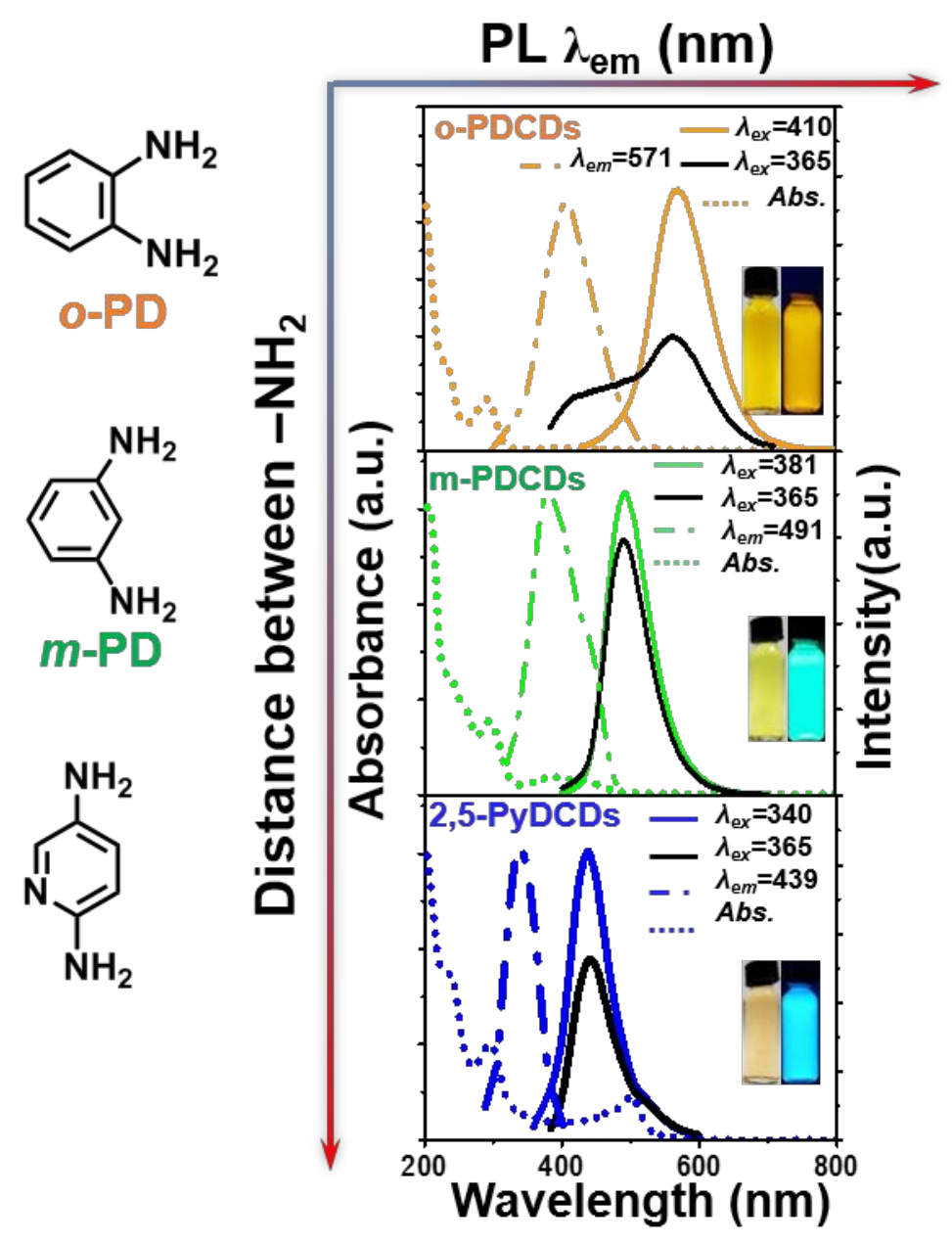

Figure S9. The emission spectrum comparison of $o$-PDCDs, $m$-PDCDs and 2,5PyDCDs, PL $\lambda_{\text {em }}$ versus the distance between two amine groups at each precursor. 